\title{
Ingresos del canon y sobrecanon petrolero y sus efectos socioeconómicos. Estudio de casos en Perúi
}

\section{Income from the oil canon and over canon and its socioeconomic effects. Case studies in Peru}

\author{
Alexci Igor Chong Ríos ${ }^{2}$ \\ Universidad Nacional Mayor de San Marcos \\ alexci.chong@unmsm.edu.pe \\ Luis Ángel Wong Valdiviezo ${ }^{3}$ \\ Pontificia Universidad Católica del Perú \\ wong.la@pucp.pe \\ German Vladimir Chong Ríos ${ }^{4}$ \\ Universidad Nacional de la Amazonía Peruana \\ vladimir.chong@gmail.com
}

Recibido: 20/03/2021 - Aceptado: 11/07/2021 - Publicado: 06/12/2021

\section{RESUMEN}

El estudio identifica los efectos del ingreso por canon y sobrecanon petrolero (CSP) sobre dos aspectos socioeconómicos: Primero, su contribución al PBI y la productividad en la región Loreto. Segundo, encontrar la misma contribución del CSP en las regiones de Loreto, Piura, Tumbes, Huánuco y Ucayali en dos dimensiones: 1) la reducción de desigualdad, medida como el gasto per cápita entre el $40 \%$ más pobre de la población y, 2) el trabajo decente, medido sobre el porcentaje de jóvenes entre 15 y 24 años que no estudian, no trabajan y no reciben capacitación. Los resultados muestran que CSP tuvieron impacto positivo, pero no significativo en el PBI y la productividad en la región Loreto. El impulso es mínimo. Representa como máximo

(C) Los autores. Este artículo es publicado por Pensamiento Crítico de la Facultad de Ciencias Económicas, Universidad Nacional Mayor de San Marcos. Este es un artículo de acceso abierto, distribuido bajo los términos de la licencia Creative Commons Atribucion - No Comercia_Compartir Igual 4.0 Internacional. (http://creativecommons.org/licenses/by-nc-sa/4.0/) que permite el uso no comercial, distribución y reproducción en cualquier medio, siempre que la obra original sea debidamente citada. 
el $9.5 \%$ de las fluctuaciones en el PBI regional y $0.13 \%$ en la productividad. En relación al segundo aspecto el efecto es positivo, significativo y exiguo sobre el gasto per cápita del 40\% más pobre. El efecto sobre el trabajo decente entre jóvenes de 15 y 24 años, de acuerdo al modelo pooled data, mostró un efecto estadístico, positivo y significativo, y en esa medida los ingresos del canon y sobrecanon no fomentaron la mejora en esta variable.

Palabras clave: Canon y sobrecanon petrolero (CSP); aspecto socioeconómico; productividad; vector autorregresivo (VAR); datos de panel.

Código JEL H2, H5, C1.

\section{ABSTRACT}

The study identifies the effects of oil canon and over-canon income (OCOC) on two socioeconomic aspects: First, its contribution to GDP and productivity in the Loreto region. Second, to find the same contribution of the OCOC in the regions of Loreto, Piura, Tumbes, Huánuco and Ucayali in two dimensions: 1) the reduction of inequality, measured as the per capita expenditure among the poorest $40 \%$ of the population and, 2) decent work, measured as the percentage of young people between 15 and 24 years of age who do not study, do not work and do not receive training. The results showed that CSP had a positive but not significant impact on GDP and productivity in the Loreto region. The momentum is minimal. It represents a maximum of $9.5 \%$ of the fluctuations in the regional GDP and $0.13 \%$ in productivity. Regarding the second aspect, the effect is positive, significant and meager on the per capita expenditure of the poorest $40 \%$. The effect on decent work among young people aged 15 and 24, according to the pooled data model, showed a statistical, positive and significant effect, and to that extent the income from the canon and over-canon did not promote improvement in this variable.

Keywords: Oil canon and over-canon (OCOC); socioeconomic aspect; productivity; vector autoregressive (VAR); panel data.

Código JEL H2, H5, C1. 


\section{Introducción}

El fundamento seminal de la asignación presupuestal de ingresos por canon y sobrecanon petrolero (CSP) decretado por Ley $\mathrm{N}^{\circ} 21678$ en el año 1976 geográficamente circunscrito inicialmente a la región Loreto, fue fomentar el desarrollo de infraestructura básica y servicios sociales. Posteriormente el alcance de los beneficios de la explotación petrolera se extendió, con leyes particulares, a las regiones de Ucayali, Piura, Tumbes y Huánuco.

La utilización de estos fondos (CSP) ha sufrido una serie de modificaciones a lo largo del tiempo. Posterior a la Ley $\mathrm{N}^{\circ} 21678$ se amplió el uso del recurso mediante Ley $\mathrm{N}^{\circ} 27506$

Los gobiernos regionales y gobiernos locales pueden utilizar los recursos del canon para el financiamiento o cofinanciamiento de proyectos u obras de infraestructura de impacto regional y local, así como para el financiamiento de Bonos Familiares Habitacionales (BFH) destinados a proyectos de vivienda del Programa Techo Propio y para el financiamiento del Programa Nacional de Vivienda Rural, mediante convenios con el Ministerio de Vivienda, Construcción y Saneamiento o el Fondo MIVIVIENDA. (El Peruano, 2018, pp 1)

La ley del presupuesto del Año Fiscal 2020 permite hasta un 20\% del uso del fondo para acciones de mantenimiento de infraestructura, así como rehabilitación afectadas por desastres naturales o antrópicos.

La distribución del fondo a las 5 regiones de estudio se distribuye de acuerdo a lo publicado por el Ministerio de Economía y Finanzas (MEF) y se resume en la tabla 1.

Aun cuando la ley autoriza la utilización del CSP para el financiamiento o cofinanciamiento de proyectos u obras de infraestructura de impacto regional o local, los indicadores económicos y sociales no han sido muy alentadores. Así, los cálculos realizados en función a los datos muestran que el PBI en Loreto durante el periodo de estudio (1994 - 2019) muestra un crecimiento de 4.3\%, mientras que el Perú creció 16.9\%. La productividad en el mismo periodo muestra un escaso crecimiento de $0.037 \%$. El 
gasto promedio del $40 \%$ de la población más pobre para las 5 regiones de estudio se incrementó en 33.95\%; y el trabajo decente, medido sobre el número de jóvenes entre 15 y 24 años que no estudian, no trabajan y no reciben capacitación en las 5 regiones de estudio disminuyó en $0.02 \%$. (Cifras calculadas de la línea de base de los principales indicadores disponibles de los objetivos de desarrollo sostenible (ODS), INEI 2018)

Tabla 1

Distribución del Canon y Sobrecanon (Porcentaje)

\begin{tabular}{|c|c|c|c|c|c|c|}
\hline \multirow[t]{2}{*}{ Organismo Púbicos } & Loreto & Ucayali & & Piura & Tumbes & $\begin{array}{c}\text { Háunuco } \\
\text { Puerto Inca }\end{array}$ \\
\hline & $\begin{array}{c}\text { Canon y } \\
\text { sobrecanon }\end{array}$ & Canon & Sobrecanon & $\begin{array}{c}\text { Canon y } \\
\text { sobrecanon }\end{array}$ & $\begin{array}{c}\text { Canon y } \\
\text { sobrecanon }\end{array}$ & Canon \\
\hline Gobierno Regional & 52 & 20 & 52 & 20 & 20 & \\
\hline Gobiernos Locales & 40 & 70 & 40 & 70 & 70 & 100 \\
\hline${ }^{*}$ Distrito productor & & 10 & & & & \\
\hline * Provincia productora & & 20 & & 20 & 20 & \\
\hline * Dpto. Productor & & 40 & & 50 & 50 & \\
\hline Universidades Nacionales & 5 & 5 & 5 & 5 & 5 & \\
\hline Inst. Inv. Amz. Peruana & 3 & 2 & 3 & & & \\
\hline inst. Sup. Tec y Pedag. & & 3 & & 5 & 5 & \\
\hline
\end{tabular}

Fuente: WEB MEF - 2020

La región Loreto durante los 26 años materia de estudio, ha recibido la suma de S/. 4,842 millones de soles por transferencia de CSP con una variabilidad anual (S/. 99.6 millones de soles). Los ingresos recibidos en el año 2019 son similares a los del año 1999. En este punto hay que destacar que el año 2016 se experimentó una caída de $68.5 \%$ con respecto al 2015 y se origina por el cierre temporal del oleoducto Nor Peruano desde mediados de febrero que imposibilitan el bombeo, además de ciertos conflictos sociales. De acuerdo al Colegio de Economistas de Loreto (2017) "el lote 192 (ex lote 1-AB) reporta una caída del $88,1 \%$; el lote 8 reporta $-42,9 \%$ y; el lote 67 con una caída de 76,3\% de producción petrolera" (p.1). La figura 1 muestra el comportamiento de los ingresos. 


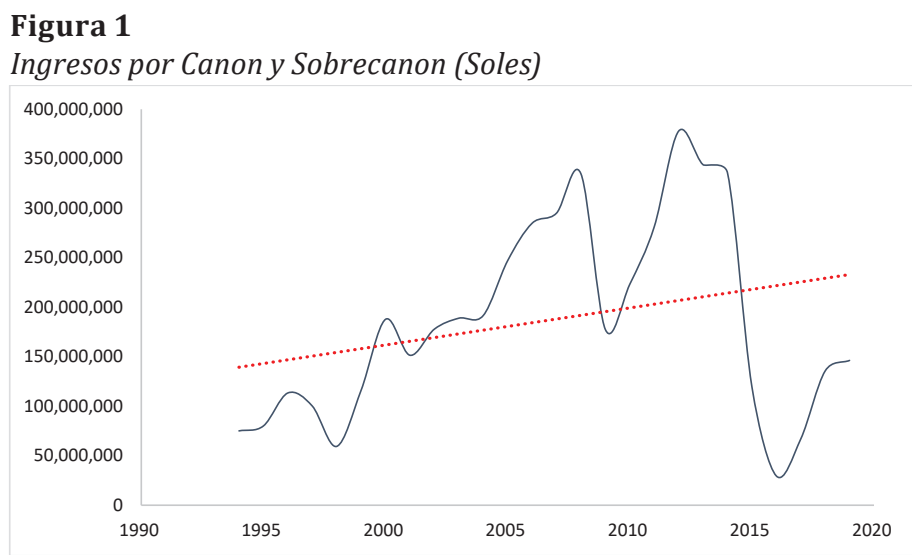

Fuente. Producción hidrocarburos - PERUPETRO - 2020

Por otro lado, las reservas probadas de petróleo son cada vez menores. Las reservas probables muestran un declive pronunciado de 411.6 en el 2004 a 101.38 millones de barriles en el 2017. Las reservas posibles son aún más desalentadoras, de 5,073 millones de barriles el 2004 desciende a 97.8 millones de barriles al 2017. La figura 2 muestra el comportamiento de las reservas probadas de petróleo.

\section{Figura 2}

Reservas Probadas de petróleo. Miles de Barriles

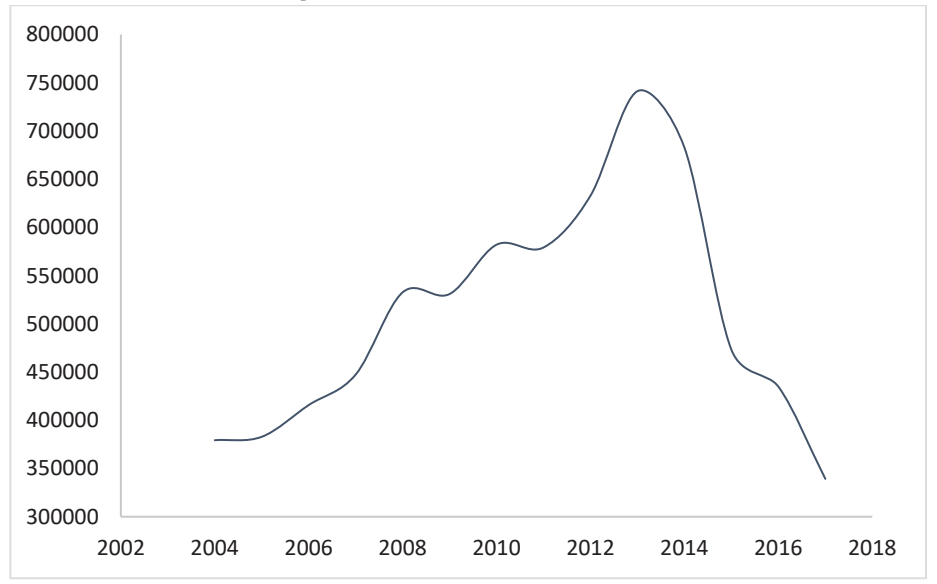

Fuente. Reservas de Hidrocarburos - PERUPETRO - 2020 
Ante las magnitudes presupuestales recibidas por CSP, el declive en las reservas probadas de petróleo que impulsan a inferir que el país ya pasó el cenit del petróleo ${ }^{5}$ y por tanto todo ello debe influir en el desempeño de los indicadores socioeconómicos, que son materia de este estudio. En esa perspectiva, nos formulamos la pregunta general de investigación ¿Cuál ha sido el efecto de los ingresos por canon y sobrecanon petrolero en algunos aspectos socioeconómicos en las regiones beneficiadas? De la pregunta general se desprenden las siguientes preguntas específicas:

1. ¿Cuál ha sido el efecto de los ingresos por canon y sobrecanon petrolero en el PBI y la productividad en la región Loreto?

2. ¿Cuál ha sido el efecto del ingreso por canon y sobrecanon petrolero en el gasto per cápita del 40\% más pobre de la población en las regiones de Loreto, Ucayali, Piura, Tumbes y Huánuco?

3. ¿El ingreso por canon y sobrecanon petrolero disminuyó la proporción de jóvenes entre 15 y 24 años de las regiones Loreto, Ucayali, Piura, Tumbes y Huánuco que no estudian, no trabajan y no reciben capacitación?

Considerando que una técnica econométrica es apropiada solo si los supuestos subyacentes detrás de esa técnica reflejan la realidad económica, se utilizó para el presente estudio un modelo de vectores autoregresivos (VAR) y un bloque de modelos de datos de panel. Los datos para el periodo 1994 - 2019 son extraídos de fuentes primarias: Banco Central de Reserva del Perú (BCRP), Perpetro, Instituto Nacional de Estadística e Informática (INEI) y del Ministerio de Economía y Finanzas (MEF)

\section{Metodología}

El enfoque del estudio es cuantitativo y el alcance descriptivo correlacional. Las hipótesis que se busca corroborar son las siguientes: 1) El ingreso por canon y sobrecanon petrolero impulsó el aumento del PBI per cápita, así como la productividad regional en Loreto. 2) El ingreso por canon y sobrecanon petrolero tuvo efecto positivo en el gasto per cápita del $40 \%$ más pobre de la población en las regiones de Loreto, Ucayali, Piura, Tumbes y Huánuco. 3) El ingreso por canon y sobrecanon 
ayudó a disminuir la proporción de jóvenes entre 15 y 24 años de las regiones Loreto, Ucayali, Piura, Tumbes y Huánuco que no estudian, no trabajan y no reciben capacitación.

Para corroborar la hipótesis 1 utilizamos un modelo Autoregresivo (VAR) con 26 datos anuales del periodo 1994 - 2019. El modelo es elegido por su capacidad de interrelacionar las diferentes variables simultáneamente, así como analizar la dinámica del tiempo.

Para la hipótesis 2 y 3 utilizamos diferentes modelos de datos de panel estacionarios, pooled panel, efectos fijos y aleatorios y un panel dinámico de Arellano y Bond que permitan contrastar y robustecer los resultados encontrados, con 11 datos anuales que corresponden al periodo 2007 - 2017.

Las variables a utilizar, así como la fuente de obtención de datos se resumen en la tabla 2, Operacionalización de las variables.

Tabla 2

Operacionalización de las variables

\begin{tabular}{|c|c|c|c|c|c|}
\hline Variable & Definición & $\begin{array}{l}\text { Tipo po su } \\
\text { naturaleza }\end{array}$ & Indicadores & $\begin{array}{l}\text { Escala de } \\
\text { medición }\end{array}$ & $\begin{array}{c}\text { Medio de } \\
\text { verificación }\end{array}$ \\
\hline $\begin{array}{c}\text { Canon y Sobrecanon } \\
\text { Petrolero }\end{array}$ & $\begin{array}{l}\text { Participación efectiva y } \\
\text { adecuada de la que gozan } \\
\text { los gobiernos regionales y } \\
\text { locales del total de los recur- } \\
\text { sos y rentas obtenidos por } \\
\text { el Estado por la explotación } \\
\text { económica del Petróleo }\end{array}$ & Cuantitativo & $\begin{array}{c}\text { Tranferencia por } \\
\text { Canon y Sobrcanon } \\
\text { Petrolero }\end{array}$ & Nominal & Perú Petro \\
\hline Productividad & $\begin{array}{c}\text { Capacidad de producción } \\
\text { por unidad de trabajo. (PBI } \\
\text { regional/Población económi- } \\
\text { camente activa) }\end{array}$ & Cuantitativo & $\begin{array}{l}\text { Cociente entre el PBI } \\
\text { regional y la PEA }\end{array}$ & Razon & $\begin{array}{c}\text { Elaboración } \\
\text { propia con } \\
\text { datos del } \\
\text { INEI }\end{array}$ \\
\hline $\begin{array}{l}\text { Gasto per capita del } \\
40 \% \text { de personas } \\
\text { mas pobres }\end{array}$ & $\begin{array}{c}\text { Gasto en soles por persona } \\
\text { que perciben el } 40 \% \text { más } \\
\text { bajo del total de la PEA }\end{array}$ & Cuantitativo & $\begin{array}{l}\text { Gasto individual en } \\
\text { soles por persona }\end{array}$ & Nominal & INEI \\
\hline $\begin{array}{l}\text { Jovenes entre } 15 \text { a } 24 \\
\text { años que no estudian, } \\
\text { no trabajan y no se } \\
\text { capacitan }\end{array}$ & $\begin{array}{c}\text { Porcentaje de la población } \\
\text { que no estudian, no trabajan } \\
\text { y no se capacitan }\end{array}$ & Cuantitativo & $\begin{array}{c}\text { Proporción de jove- } \\
\text { nes que no estudian, } \\
\text { no trabajan y no se } \\
\text { capacitan }\end{array}$ & Nominal & INEI \\
\hline Precio del petroleo & $\begin{array}{l}\text { Precio de mercado del barril } \\
\text { de petróleo }\end{array}$ & Cuantitativo & $\begin{array}{l}\text { Precio internacional } \\
\text { del barril de petróleo }\end{array}$ & Nominal & STATISTA \\
\hline
\end{tabular}

Elaboración propia 


\subsection{El Modelo}

2.1.1. Modelo de Vectores Autorregresivos (VAR)

De acuerdo a Novales (2017) "El modelo VAR permite caracterizar las interacciones simultáneas multivariantes. No especifica variables exógenas y endógenas y expresa un sistema de ecuaciones de forma reducida sin restringir. El modelo no impone restricciones al modelo estructural descrito, por lo que no se incurre en errores de especificación” (p.2)

Utilizamos dos modelos VAR a fin de determinar, por un lado, el efecto del CSP en el PBI de Loreto y la productividad y, por otro lado, los efectos diferenciales del CSP por niveles de gobierno (regional y local). No obstante, en vista de los choques del precio del barril del petróleo a nivel internacional traducido en disminución en las transferencias por CSP, así como la paralización de la producción petrolera por problemas sociales, utilizamos una variable binaria que separa el análisis en dos periodos: 1: Periodo 1994 - 2012, 0: Periodo 2013 - 2019.

La correlación entre el precio del barril del petróleo y la transferencia por canon y sobrecanon es de 0.82 . El comportamiento es bastante similar en ambas series. La figura 3 muestra lo indicado.

\section{Figura 3}

Evolución ingresos por canon y sobrecanon y precio del petróleo
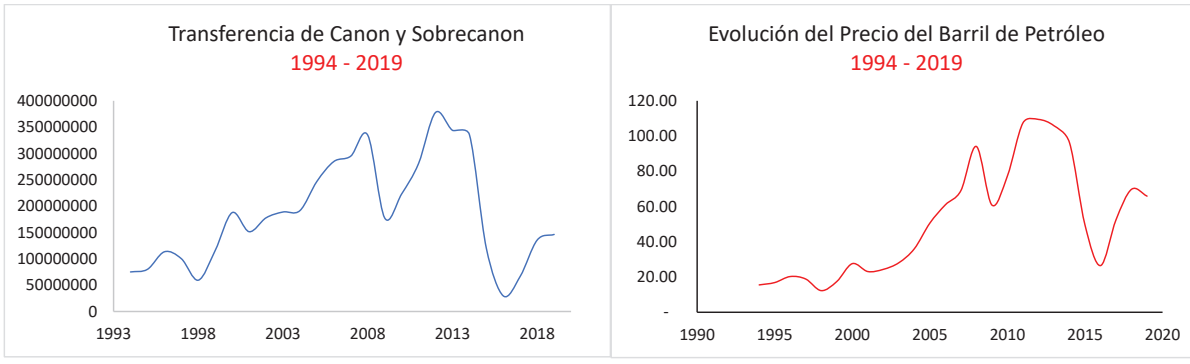

Elaboración propia.

Por tanto, la tendencia entre ambas series es similar. La figura 4 muestra el comportamiento de las tendencias para ambos periodos, en el precio del barril de petróleo. 


\section{Figura 4}

Tendencias del Precio del Barril del Petróleo

Evolución del Precio del Barril de Petróleo 2013 - 2019

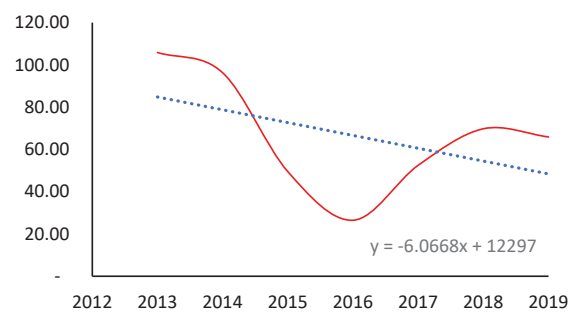

Evolución del Precio del Barril de Petróleo 1994 - 2012

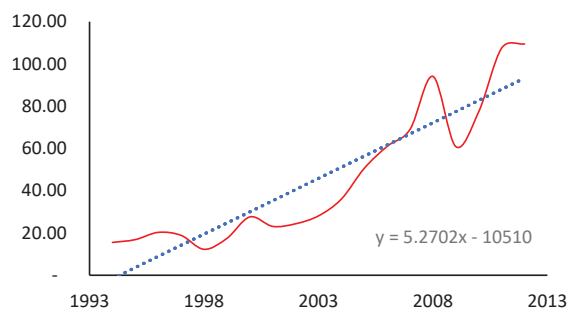

Elaboración propia

Con la definición de los cambios tendenciales en el periodo de estudio el primer modelo VAR es el siguiente.

$$
\begin{gathered}
\text { PBI }_{t}=\psi+\sum_{i=1}^{k} \beta_{i} \text { PBI }_{t-i}+\sum_{i=1}^{k} \beta_{i} \text { PROD }_{t-i}+\sum_{j=1}^{k} \beta_{j} \text { CANON }_{t-j}+ \\
\sum_{m=1}^{k} D m+u_{1 t}
\end{gathered}
$$

PBIr : : PBI región Loreto

PROD : Productividad

CANON : Canon y sobrecanon petrolero

Dm ～: Dicotómica. 1: $1994-2012$

$$
0: 2013-2019
$$

$u_{t} \quad$ : Choques (impulsos o innovaciones)

El modelo espera encontrar signo positivo y significativo del coeficiente CSP hacia el PBI regional (PBIr) y la productividad (PROD). Se espera que la variable dicotómica $(\mathrm{Dm})$ muestre signo positivo y significativo ya que ésta representa las transferencias del canon y sobrecanon petrolero en el periodo 1994 - 2012 (pendiente positiva).

El segundo modelo busca determinar el efecto del canon y sobrecanon petrolero por nivel de gobierno sobre el PBI regional y la productividad. Al 
igual que el modelo anterior se espera la misma dirección de los signos y significancia estadística. El modelo 2 se ilustra a continuación.

$$
\begin{aligned}
& P B I r_{t}=\psi+ \\
& \sum_{i=1}^{k} \beta_{i} P B I r_{t-i}+\sum_{i=1}^{k} \beta_{i} \text { PROD }_{t-i}+\sum_{j=1}^{k} \beta_{j} \text { CANREG }_{t-j}+\sum_{j=1}^{k} \beta_{j} \text { CANLOC }_{t-j}+u_{1 t} \\
& \text { PBIr : PBI regional } \\
& \text { PROD : Productividad } \\
& \text { CANREG : Canon y sobrecanon petrolero asignado al Gobierno Regional } \\
& \text { CANLOC : Canon y sobrecanon petrolero asignado a los Gobiernos } \\
& \text { Locales } \\
& u_{t} \quad: \text { Choques (innovaciones) }
\end{aligned}
$$

Analizamos la presencia de estacionariedad de las variables para definir si modelamos el modelo VAR en niveles o en primeras diferencias. El test de Dickey Fuller Aumentada (DFA) indica estacionariedad en primeras diferencias I(1). (Ver Anexo 1). La tabla 3 muestra los test de raíz unitaria y la figura 5 muestra las series en niveles y primeras diferencias.

Tabla 3

Prueba de Dickey Fuller en nivel y primeras diferencias

\begin{tabular}{lcc}
\hline \multirow{2}{*}{ Variables } & Dickey Fuller Aumentada & Dickey Fuller Aumentada \\
\cline { 2 - 3 } & Level & (First Difference) \\
\cline { 2 - 3 } & Con intercepto & Con intercepto \\
\hline PBIr & 0.9306 & 0.0304 \\
Canon y sobrecanon & 0.2889 & 0.0039 \\
Productividad & 0.5328 & 0.0360 \\
\hline
\end{tabular}

Elaboración propia

Un aspecto neurálgico en la estimación mediante el VAR es seleccionar la longitud apropiada del rezago. Los distintos criterios muestran mantener un rezago de acuerdo al modelo VAR. (Anexo 2).

\section{Figura 5}


Variables en niveles y primeras diferencias. 1994 - 2019
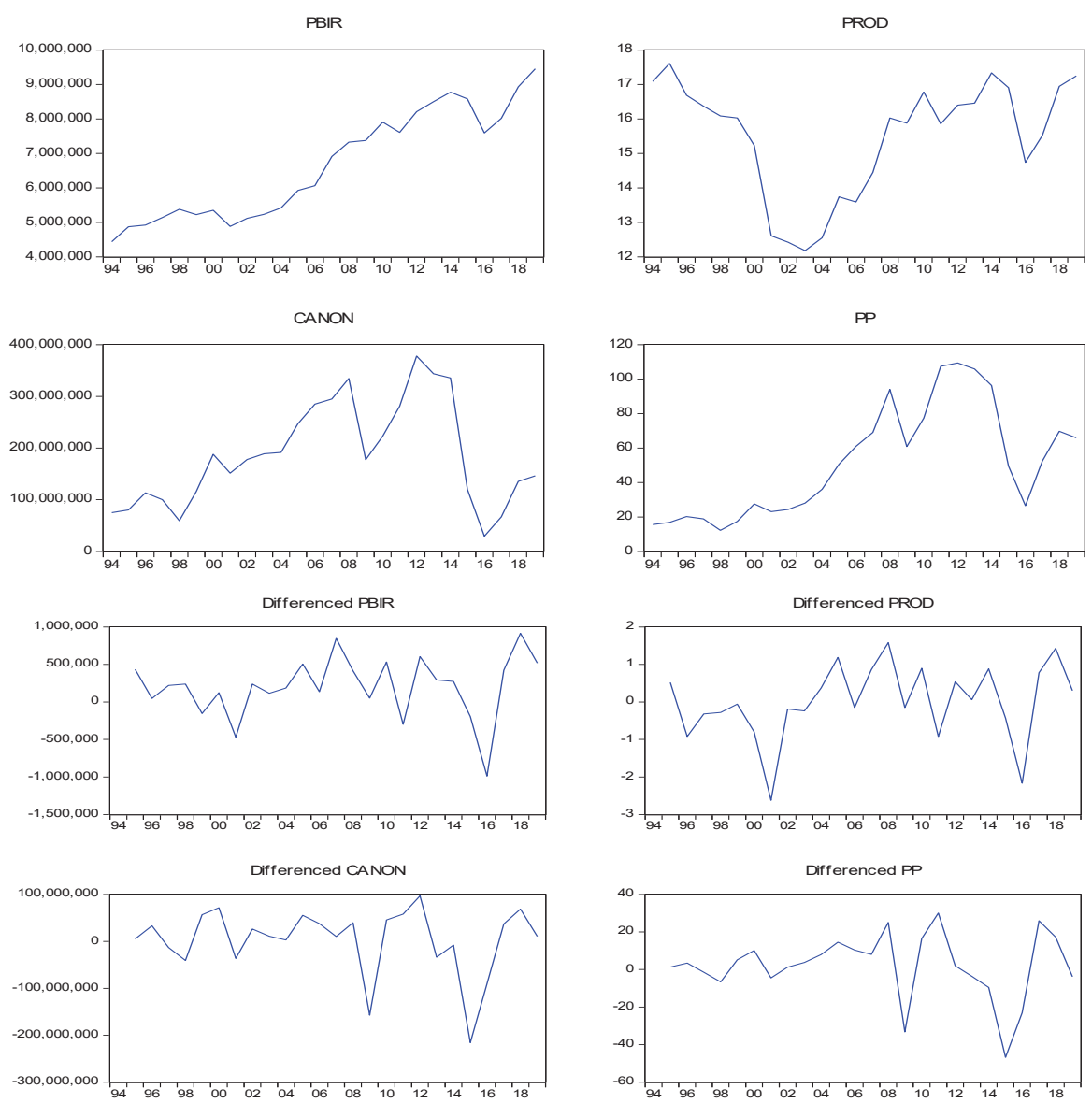

Elaboración propia. Eviews

El análisis impulso respuesta a realizar permite determinar la perturbación en una innovación en el modelo VAR y como ésta crea una reacción en cadena en todas las variables mediante la estructura dinámica del modelo. El tipo de evolución en el valor de cada variable nos permitirá determinar si los choques son transitorios o permanentes. El análisis se complementa con la descomposición de varianza que permite cuantificar el choque en una desviación estándar del ingreso por CSP sobre las variaciones (fluctuaciones) en las variables dependientes. 
Finalmente, se realiza una serie de pruebas para ver que el modelo cumpla su correcta especificación. Las pruebas de diagnóstico fueron enfocadas en tres posibles problemas: Autocorrelación, Normalidad y Prueba de estabilidad de los parámetros.

2.1.2. Modelo de datos de panel

Para comprobar la hipótesis 2 y 3 realizamos dos bloques de modelos de panel por separado y comprobamos si la presencia de los ingresos por CSP en las 5 regiones tienen impactos sobre el gasto per cápita entre al 40\% de la población más pobre y sobre el porcentaje de jóvenes entre 15 y 24 que no estudian, no trabajan y no reciben capacitación. Aplicamos distintos modelos de datos de panel estacionarios como son: Datos agrupados, efectos fijos y aleatorios. Para robustecer los resultados y para efectos comparativos, aplicamos una estimación dinámica en diferencias GMM de Arellano y Bond. La idea es comparar resultados, corroborar la dirección del signo de las regresiones de panel y las significancias estadísticas.

Los datos serán tratados en sus niveles originales a fin de aprovechar la información de comovimientos de los datos. El modelo de datos de panel estacionario a estimar es el siguiente-e.

$$
\begin{gathered}
G P K M P_{i t}=\alpha+\beta C S P_{i t}+\psi t_{i t}+\boldsymbol{\mu}_{i i}+\varepsilon_{i t} \\
N E N T N C_{i t}=\alpha+\beta C S P_{i t}+\psi t_{i t}+\boldsymbol{\mu}_{i \boldsymbol{i}}+\varepsilon_{i t}
\end{gathered}
$$

GPKMP $_{i t} \quad$ : Gasto per cápita entre al $40 \%$ de la población más pobre NENTNC $_{i t}$ : Jóvenes entre 15 a 24 años que no estudian, no trabajan y no se capacitan.

$\alpha$ : Intercepto

$\beta$ : Coeficiente de pendiente de $\operatorname{CSP}_{\mathrm{it}}$

CSP $_{\text {it }}$ : Canon y sobrecanon petrolero

$\psi$ : Coeficiente de pendiente de $t_{i t}$

$t \quad$ : Tiempo

$\mu_{i}$ : Efecto individual específico 
$\mathcal{E}$ : Término de error $i$ en el tiempo $t$.

Para el modelo con variable endógena $G P K M P_{i t}$ se espera signo positivo y significativo del canon y sobre canon petrolero (CSP) de tal manera que los fondos asignados ejerzan un efecto de aumento en el gasto per cápita entre el $40 \%$ de la población más pobre. Para el modelo que considera la variable endógena $N E N T N C_{i t}$ se espera que la variable CSP muestre signo negativo y significativo ya que esto se interpretará que el aumento en los fondos del CSP reduce el porcentaje de jóvenes que no estudian, no trabajan y no reciben capacitación.

El test de Pasaran CD es usado para verificar si los residuos están autocorelacionados a través de la dependencia transversal entre sujetos y puede conducir a sesgos en los resultados de las pruebas (también llamado correlación contemporánea). La hipótesis nula (Ho) sostiene que los residuos no están correlacionados transversalmente. En relación a la heteroscedasticidad, el test de Wald modificado será utilizado para verificar varianza constante en el modelo de efectos fijos.

El modelo dinámico Arellano y Bond es el siguiente.

$$
G P K M P_{i t}=\alpha+\beta G P K M P_{i t-1}+\delta C S P_{i t}+\mu_{i}+\varepsilon_{i t}
$$

GPKMP $_{i t} \quad$ : Gasto per cápita entre al $40 \%$ de la población más pobre $\alpha \quad$ : Intercepto

$\beta \quad$ : Coeficiente de pendiente (efecto de corto plazo de GPKMP ${ }_{i t-1}$ )

CSP $_{\text {it }} \quad:$ Canon y sobrecanon petrolero

$\delta \quad:$ : Coeficiente de pendiente de $\mathrm{CS}_{i t}$

$\mu_{i} \quad$ : Efecto individual específico

$\varepsilon \quad$ : Término de error $i$ en el tiempo $t$.

$$
N E N T N C_{i t}=\alpha+\beta N E N T N C_{i t-1}+\delta C S P_{i t}+\boldsymbol{\mu}_{i}+\varepsilon_{i t}
$$

$N E N T N C_{i t}$ : Porcentaje de Jóvenes entre 15 y 24 que no estudian, no trabajan y no reciben capacitación. 
$\alpha$ : Intercepto

$\beta$ : Coeficiente de pendiente (efecto de corto plazo de $N E N T N C_{i t-1}$ )

$\operatorname{CSP}_{\text {it }}$ : Canon y sobrecanon petrolero

$\delta$ : Coeficiente de pendiente de $\mathrm{CS}_{i t}$

$\mu_{i}:$ Efecto individual específico

$\mathcal{E}$ : Término de error $i$ en el tiempo $t$.

Los signos y significancia estadística esperada para el modelo dinámico son los mismos para los modelos estáticos.

Para el caso de la Autocorrelación en la estimación dinámica se aplica el test de autocorrelación de Arellano y Bond, el cual sostiene como hipótesis nula Ho que no existe autocorrelación, a un nivel $\operatorname{Pr}>0.05$.

\section{Marco Teorico}

\subsection{Antecedentes}

Correa y Morocho (2016) analizaron el impacto del CSP en la región Piura para el periodo 1984 - 2014. Mediante el uso de un modelo VAR y datos de panel encuentran que en términos generales el impacto ha sido positivo orientado más al corto que al largo plazo. Desagregando entre el impacto generado por el gobierno regional y local sobre la actividad económica encuentra que los resultados del canon del gobierno regional no son estadísticamente significativos en contraste con los del gobierno local que son de mayor impacto y significativos con un impulso de hasta por 1.5 años. El impacto social del canon sobre el índice de desarrollo humano (IDH) es negativo. Sin embargo, encuentran resultados positivos en distritos urbanos y productores, por lo que se infiere que el impacto se focaliza en áreas menos vulnerables.

Orihuela (2014) realizó un estudio para la región Loreto enmarcado en el periodo 1994 - 2012 con el objetivo de verificar el valor y tendencia de la riqueza e inferir si existió inversión genuina, por consiguiente, dirección 
hacia el desarrollo sostenible. Los resultados de la investigación utilizando un indicador compuesto de inversión genuina, mostró que la riqueza per cápita se incrementó en 13\% lo que permitió concluir que la región Loreto estuvo en la senda del desarrollo, a pesar de ser una región pobre y desigual. Sin embargo, precisa que aunque el crecimiento de la riqueza per cápita aumentó durante el periodo de estudio no garantiza que este crecimiento se mantenga debido a la poca evidencia del capital humano e institucional en la generación de riqueza, así como acertadas decisiones de política local. Las reservas probadas de petróleo que conforman el capital natural se proyecta a solo 11 años de acuerdo al Ministerio de Energía y Minas (MEM, 2010 p.13). Finalmente, el crecimiento del PBI per cápita (33\%) mayor al crecimiento de la riqueza per cápita (13\%) en el período 1994-2012, demuestra que no se ha estado acumulando suficiente capital, premisa determinante para asegurar desarrollo sostenible.

Clancy y kerremans (2016) realizaron un estudio encargado por el Instituto Chaikuni sobre la dependencia petrolera en la Amazonia Peruana con testimonio del pueblo nativo Kichwa del río tigre. Con más de 40 años de explotación petrolera y 500,000 hectáreas, la contaminación ha devastado cuatro cuencas de los principales afluentes del rio Amazonas con tuberías de petróleos corroídas y con fugas que contaminaron los suelos. Las tierras no producen como antes y encontraron metales tóxicos en los animales de los alrededores. Asimismo, expresan que la empresa Pluspetrol con la empresa China CPNC declaran una política de responsabilidad ambiental y social. Sin embargo, sus comportamientos manifiestan todo lo contrario por la renuencia de remediar los pasivos ambientales originados, así como los acuerdos de apoyo comunal contraídos.

Labarthe (2019) investigador por la Asociación de Contribuyentes del Perú, en una nota periodística en el diario La República, señala que la región Piura recibe un promedio histórico del 52\% del canon que se distribuye a nivel nacional. Sin embargo, al revisar la ejecución del 2016 al 2018 cerca del $43 \%$ de lo asignado se gastó sin ningún proyecto asignado. Los gastos se dirigieron básicamente a compras de insumos, carburantes y afines, así como a mantenimiento de infraestructura pública, inversiones que no sirven para generar desarrollo productivo o dar un valor agregado a la calidad de vida de la población. Enfatiza que se observa una tendencia de gastos en pequeños proyectos y que no se estaría cumpliendo con 
la Ley de Canon, que señala que los recursos otorgados a los gobiernos regionales y locales se usen exclusivamente en obras de infraestructura productiva de impacto regional y local.

Perry y Olivera (2009) en un estudio para Colombia buscan determinar el impacto de las regalías mineras y petroleras (entre otros objetivos) sobre el desarrollo económico (en términos de PBI per cápita) en los municipios y departamentos donde se ubica la explotación. El estudio encuentra impacto positivo y significativo de las regalías por hidrocarburos en los municipios, pero negativos en los departamentos. Los autores señalan "Es posible que éstas diferencias se deban a que los efectos de "maldición" de los recursos naturales predominan en aquellas entidades territoriales que dependen excesivamente del petróleo, ya que, de una parte, las regalías del petróleo son mucho mayores que las del carbón y generan más esfuerzos por capturarlas y, de otra parte, la producción petrolera genera menos encadenamientos hacia atrás y hacia delante en comparación con el caso de la pequeña y mediana minería del carbón". (p. 51). Finalmente, se aplica estudios de casos para ver si la calidad de las instituciones determina el impacto de la abundancia de recursos sobre el crecimiento.

\subsection{Aspectos teóricos}

Enfocamos el desarrollo del aspecto teórico en dos posiciones: Los efectos negativos de los recursos naturales y los efectos positivos que estos conllevan.

\subsubsection{Efectos Negativos}

La literatura señala que en la década de los 70's y 80's Alan Gelb y Richard Auty fueron los primeros en analizar de un modo teórico, mas no basado en evidencia empírica, la falla de países liderados por recursos naturales y sugieren que muchos de los factores económicos y políticos jugaron un rol en la baja performance de estas economías. Acuñaron el término "maldición" para referirse a la asociación entre la dependencia extractiva de una determinada región al pobre desempeño económico y bajo grado de desarrollo institucional.

Sachs y Warner (1995) desarrollaron de un modo prolífico el tema señalando que una de las características sorprendentes del crecimiento 
económico es que las economías con abundantes recursos naturales han tendido a crecer con menor rapidez que las economías con escasos recursos naturales. La teoría es sostenida mediante un estudio empírico a 97 países en vías de desarrollo mediante la tasa de crecimiento anual de cada país para el periodo 1970 - 1989 en relación a las exportaciones de 1970 basados en recursos naturales por país, medido como porcentaje del PBI. Un análisis a priori indica que en promedio, los países con un alto valor de exportaciones basado en sus recursos tienden a tener más baja tasa de crecimiento $^{6}$. Mediante datos de panel el estudio encuentra evidencia de una relación negativa entre intensidad de recursos naturales y crecimiento refutando la hipótesis de que un auge en los recursos naturales puede servir como impulso al crecimiento de largo plazo y se comprobó de manera contraria que la tasa nominal de crecimiento disminuye una vez que el auge de los recursos naturales pasaba.

De acuerdo a Frankel (2012) citados por Reina, Alejo y Devia (2018) existen seis hipótesis que explican por qué los recursos naturales se vuelven una maldición, destacando que la volatilidad en los precios de los commodities e instituciones anárquicas apalanca el decrecimiento económico.

Otro aspecto relacionado a los efectos negativos es la denominada enfermedad holandesa. De acuerdo a Ravago y Roumasset (2015), Lanteri (2016) el término fue acuñada por la revista inglesa "The Economist" para explicar el declive de la industria manufacturera de los Países Bajos (Holanda) al descubrir gas natural en la década de 1960. Posteriormente el término se expandió para incluir hidrocarburos, agricultura y minerales. El término refiere a un efecto adverso sobre la manufactura debido a descubrimientos en recursos naturales. Específicamente, cuando un país experimenta un auge de recursos debido a un descubrimiento de recursos transables y/o a un aumento en el precio de los recursos, normalmente experimenta una apreciación real de su tipo de cambio y, como resultado del aumento de los salarios, una reubicación de algunas de las fuerzas laborales al sector de recursos transables. Una apreciación real reduce la competitividad internacional de otros sectores transables porque las exportaciones basadas en recursos naturales desplazan otros productos basados en manufactura ${ }^{7}$. El país enfrenta el riesgo de un proceso de desindustrialización. Este fenómeno conocido como "enfermedad holandesa" 
llamó la atención en los años 50's cuando el gas natural descubierto en Países Bajos (Holanda) eventualmente golpeó la competitividad del sector manufacturero holandés ${ }^{8}$.

El modelo de la trampa de la grapa (staple grap) de acuerdo a Reina, Alejo y Devia (2018) consiste en "explotar un recurso natural determinado bajo un marco institucional favorable al despilfarro, la pereza fiscal y de los empresarios, especialmente industriales, que resultan beneficiados de medidas proteccionistas" (p. 3).

Citando a Auty y Gelb (2000) los autores señalan

Según el modelo de la trampa de la grapa, los países pobres en recursos naturales tienden a desarrollar estados 'desarrollistas', con una serie de condiciones institucionales que los ayudan a un crecimiento rápido, sostenido y equitativo. De ser emulados por los países ricos en recursos naturales, tal tendencia podría ayudarlos a no caer en la trampa del crecimiento grampeado. $(p, 15)$

\subsubsection{Efectos positivos}

La staple theory de acuerdo a Domínguez (2009) "fue desarrollada por el economista e historiador canadiense Harold Innis entre 1930 y 1940 . Innis describió un proceso de crecimiento extensivo de la producción, mediante la puesta en explotación de recursos naturales por trabajadores inmigrados. Se trataba de producir bienes básicos (pieles, bacalao, trigo, madera) con salida en los mercados internacionales, lo que requirió, a partir de un determinado momento, reducir costes de transporte" (p. 423)

Por tanto, del uso extensivo en el uso de los recursos naturales se genera un crecimiento intensivo que contrarrestará los rendimientos decrecientes gracias al progreso tecnológico. Tello (2013) señala que

"el sector de exportación intensivo genera: (i) eslabonamientos hacia adelante (si los productos de los sectores base/principal se usan como insumos para otras industrias) y hacia atrás, por la demanda de insumos y bienes de capital; (ii) incremento de la demanda de bienes de consumo final derivado de los trabajadores empleados en los centros de explotación y producción de los RRNN; y (iii) eslabonamientos fiscales por la recaudación proveniente de los ingresos 
de los sectores intensivos en RRNN, los cuales inciden en el crecimiento de los sectores no principales o no base y, consecuentemente, en el crecimiento de las regiones donde se localizan tales RRNN. Si los productos del sector principal/base son de exportación, la demanda de inversión y la clase empresarial pueden proceder del exterior. Es decir, no se limitan a los recursos y ahorros internos de las regiones". (p. 43)

La teoría de la base económica de acuerdo a Tello, 2006) sostiene que el desarrollo de una región se basa en las interacciones de actividades básicas con otras regiones que impulsan el desarrollo de actividades no básicas. El sostén fundamental son las importaciones que generan otras regiones de las actividades básicas producidas en la región. Por tanto, factores internos como abundancia de recursos, producción y distancia determinan el crecimiento regional (p.37).

\section{Resultados}

\subsection{Hipótesis 1. Aplicando Modelo VAR}

La tabla 4 muestra las estadísticas descriptivas de cada variable.

Tabla 4

Estadísticas descriptivas de las variables. 1994 - 2019

\begin{tabular}{lccccc}
\hline Variable & Obs & Mean & Std. Dev. & Min & Max \\
\hline PBIr & 26 & 6660282 & 1576119 & 4439239 & 9453333 \\
PRODUCT & 26 & 15.5 & 1.655295 & 12 & 18 \\
CANON & 26 & $1.86 \mathrm{E}+08$ & $9.95 \mathrm{E}+07$ & $2.96 \mathrm{E}+07$ & $3.78 \mathrm{E}+08$ \\
CANREG & 26 & $1.06 \mathrm{E}+08$ & $6.02 \mathrm{E}+07$ & $1.95 \mathrm{E}+07$ & $2.43 \mathrm{E}+08$ \\
CANLOC & 26 & $6.66 \mathrm{E}+07$ & $3.54 \mathrm{E}+07$ & 8403269 & $1.34 \mathrm{E}+08$ \\
DICOT & 26 & 0.7307692 & 0.4523443 & 0 & 1 \\
\hline
\end{tabular}

Elaboración propia

Los resultados de la estimación del primer modelo VAR se muestran en la tabla 5. Los ingresos por CSP tienen un efecto positivo y no significativo sobre el PBI regional y la productividad. El efecto sobre el PBI regional y la productividad correspondiente al periodo $1994-2012$ no 
tuvo impacto positivo ni significativo. Sin embargo, en el periodo 2013 - 2019 si hubo impacto positivo, pero estadísticamente no significativo tanto para el PBI regional como para la productividad. Esta aparente paradoja de efectos negativos en las variables de interés (PBI regional y productividad) cuando aumentan los ingresos por CSP y efectos positivos en las mismas variables de interés cuando disminuyen los ingresos por CSP podemos aproximarlos con las siguientes explicaciones. Primero, los resultados aunque señalan signo positivo, no son estadísticamente significativos ${ }^{9}$. Segundo, las magnitudes mostradas en el rezago de la variable $D_{C A N O N_{-1}}$ la cual señala la diferencia entre periodos $\left(t-t_{-1}\right)$, es una magnitud muy pequeña, lo que evidencia la escasa participación del ingreso por CSP en el PBI regional y la productividad. Por tanto, la participación del CSP como variable de impulso a una mejora en las variables de interés, es prácticamente nula.

Tabla 5

Estimación modelo VAR

\begin{tabular}{|lccc|}
\hline & DPBIR & DPROD & DCANON \\
\hline DPBIR(-1) & -0.047358 & $1.46 \mathrm{E}-07$ & 32.21327 \\
& -0.4561 & $-1.20 \mathrm{E}-06$ & -77.142 \\
DPROD(-1) & -34072.79 & 0.044139 & -29266436 \\
& -177396 & -0.45745 & $-3.00 \mathrm{E}+07$ \\
DCANON(-1) & 0.002192 & $4.84 \mathrm{E}-10$ & 0.132071 \\
& -0.00143 & $-3.70 \mathrm{E}-09$ & -0.24266 \\
C & 237085.7 & 0.096918 & -31887986 \\
& -181302 & -0.46752 & $-3.10 \mathrm{E}+07$ \\
D1 & -60860.44 & -0.198011 & 39680652 \\
& -202131 & -0.52123 & $-3.40 \mathrm{E}+07$ \\
\hline
\end{tabular}

Fuente Elaboración propia

() Error estándar

${ }^{* * *} \mathrm{p}<0.01{ }^{* *} \mathrm{p}<0.05{ }^{*} \mathrm{p}<0.1$

La figura 6 muestra que la función impulso respuesta permite identificar la respuesta de las variables PBI regional y productividad cuando un choque del ingreso por CSP es capturado por el termino de error ${ }^{10}$. En relación al efecto del CSP sobre el PBI regional, el efecto positivo generado 
en el primer año cae bruscamente al siguiente año, reportando choques pequeños en los años 3 y 4, para posteriormente no mostrar efecto alguno en los siguientes periodos. En relación al choque sobre la productividad ésta también sufre un declive brusco, pero en un periodo de dos años, para posteriormente mostrar en los siguientes dos años declives cortos hasta alcanzar su valor de estado estable, donde permanece sin efecto alguno sobre la productividad. Complementariamente, el análisis de descomposición de varianza indica que el choque del ingreso por CSP representa fluctuaciones constantes en el tiempo de $9.48 \%$ en el PBI regional. En relación al choque en productividad el segundo año representa el $0.07 \%$ para posteriormente causar de forma constante $0.13 \%$. La magnitud del CSP representa variaciones porcentuales pequeñas en el PBI regional y la productividad. El mayor impulso se da en un $90 \%$ en la misma variable rezagada. Anexo (3)

\section{Figura 6}

Respuesta del PBI regional y Productividad ante un choque del CSP

Response to Cholesky One S.D. Innovations Response of DPBIR to DCANON

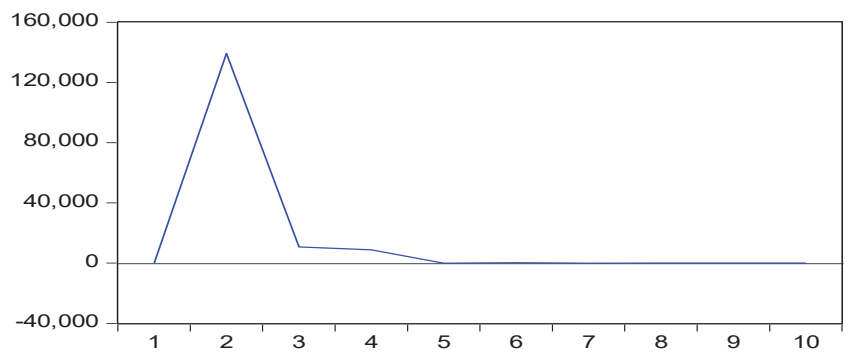

Response of DPROD to DCANON

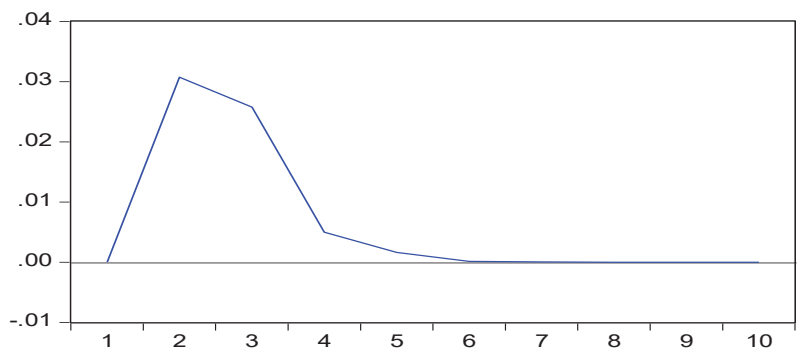

Elaborado en Eviews 
El diagnóstico de violación a los supuestos muestra que el modelo no muestra autocorrelación. El análisis de correlograma muestra que los residuos se encuentran dentro de la banda establecida con valores de DW por cada modelo de 2.04, 1.91 y 1.86. (Anexo 4). El test de Normalidad muestra una distribución con simetrías y curtosis entre 0 y 3 y un JB significativo (Anexo 5). Por lo que el modelo refleja el comportamiento real de las variables en el periodo de estudio.

En relación al efecto del CSP desagregado por niveles de gobierno (regional y local) se efectuó el segundo modelo VAR. El modelo mostró ausencia de autocorrelación y estabilidad en los parámetros considerando 1 rezago. El efecto del CSP del gobierno regional sobre el PBI regional y la productividad es positivo, pero no significativo. Mientras que el efecto del CSP de los gobiernos locales, para ambos casos es negativo y no significativo. La tabla 6 muestra los resultados.

Tabla 6

Estimación segundo modelo VAR

\begin{tabular}{lcccc}
\hline & D(PBIR) & D(PROD) & D(CANREG) & D(CANLOC) \\
\hline D(PBIR(-1)) & -0.104783 & $7.88 \mathrm{E}-08$ & 0.044866 & 21.8923 \\
& -0.45399 & $-1.20 \mathrm{E}-06$ & -43.1396 & -29.6608 \\
D(PROD(-1)) & -23605.47 & 0.076697 & -14493510 & -17556016 \\
& -172547 & -0.45093 & $-1.60 \mathrm{E}+07$ & $-1.10 \mathrm{E}+07$ \\
D(CANREG(-1)) & 0.005728 & $2.23 \mathrm{E}-09$ & 0.826939 & 0.450903 \\
& -0.0054 & $-1.40 \mathrm{E}-08$ & -0.51269 & -0.3525 \\
D(CANLOC(-1)) & -0.002438 & $-2.62 \mathrm{E}-09$ & -0.902141 & -0.381207 \\
& -0.00729 & $-1.90 \mathrm{E}-08$ & -0.69258 & -0.47619 \\
C & 200280.9 & -0.032439 & 633681 & -4202965 \\
\hline
\end{tabular}

Fuente Elaboración propia

() Error estándar

$* * * \mathrm{p}<0.01^{* *} \mathrm{p}<0.05^{*} \mathrm{p}<0.1$

En relación al análisis impulso respuesta el comportamiento se muestra en la figura 7 y es el siguiente: 
Figura 7

Respuesta del PBI regional y Productividad ante un choque del CSP Regional y Local, 2007 - 2017

Response to Cholesky One S.D. Innovations
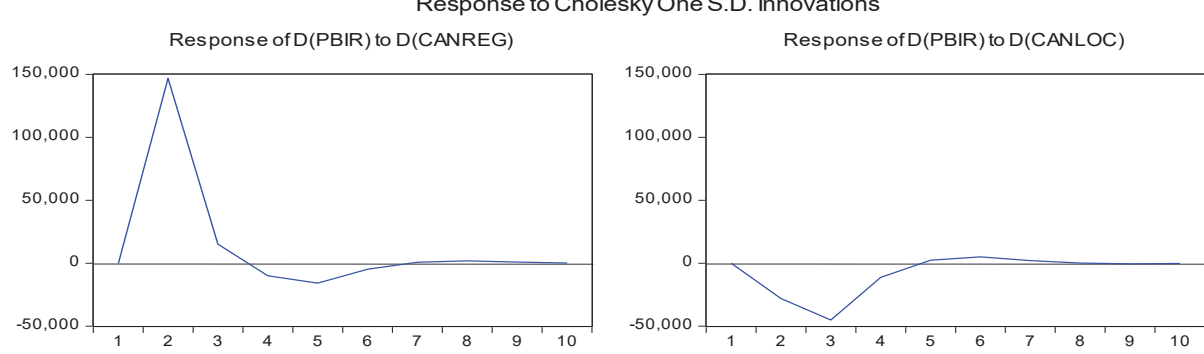

Response of D(PROD) to D(CANREG)
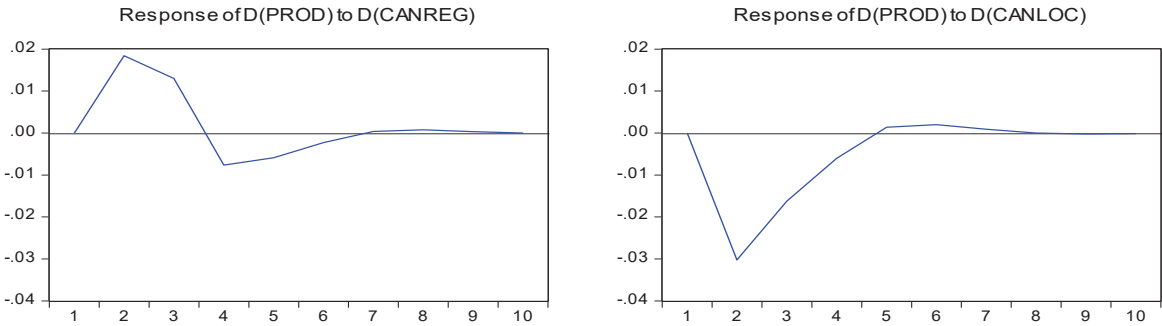

Elaboración propia.

Efecto del Canon Regional. Un choque de una desviación estándar al PBI regional, ésta inicialmente se incrementa. Sin embargo, esta respuesta positiva cae rápidamente en el segundo año cuando alcanza su valor de estado estable, desde donde desciende a la región negativa en el 4to, 5 to y 6 to año hasta volver a alcanzar el estado estacionario. Con respecto a la productividad el efecto es positivo en los primeros tres años descendiendo a la zona negativa hasta el 6 to año para posteriormente no ejercer efecto alguno.

Efecto del Canon Municipal. La respuesta del PBI regional y la productividad al CSP municipal es negativa en los primeros 5 años para posteriormente el 6to año manifestar un ligero aumento y permanecer en estado estacionario el resto de períodos.

La descomposición de varianza muestra el porcentaje de variación inesperada en cada variable producida por choques en otras variables. De tal forma que permite evaluar la importancia económica de este impacto como porcentaje. El CSP regional representa el 11\% de las variaciones de las fluctuaciones en el PBI regional y el $0.05 \%$ en la productividad. Mientras que 
el CSP local representa el $1.4 \%$ y el $0.1 \%$ en el PBI regional y productividad respectivamente. (Anexo 6)

El modelo presenta no autocorrelación con valores DW 2.04 y 1.9. El test de normalidad no sigue una distribución normal multivariada. No obstante, Arias y Torres (2004) citando a Fernández-Corugedo (2003) argumentan que es más importante que el VAR cumpla con la prueba de errores no autocorelacionado que con la normalidad multivariada.

\subsection{Hipótesis 2 y 3. Aplicando Datos de Panel}

La tabla 7 muestra las estadísticas descriptivas de los datos. Como se observa es un panel balanceado debido a que los datos son completos para cada variable. Existe una alta variabilidad en el gasto per cápita del $40 \%$ más pobre entre las distintas regiones de estudio (43\%). La proporción de jóvenes que no estudian, no trabajan y no se capacitan, entre las regiones y dentro de las regiones se encuentran en un rango mínimo de 10 puntos (overall). Así mismo, la variabilidad de ingresos por CSP entre las regiones y dentro de las regiones por año muestra alta variabilidad $\left(1.64 \times 10^{8}\right)$.

Tabla 7

Estadísticas descriptivas de Datos de Panel

2007 - 2017

\begin{tabular}{|c|c|c|c|c|c|c|c|}
\hline \multicolumn{2}{|l|}{ Variable } & \multirow{2}{*}{$\begin{array}{l}\text { Mean } \\
307.2182\end{array}$} & \multirow{2}{*}{$\begin{array}{r}\text { Std. Dev. } \\
53.64689\end{array}$} & \multirow{2}{*}{$\begin{array}{ll}\text { Min } & \\
208\end{array}$} & \multirow{2}{*}{$\begin{array}{l}\text { Max } \\
412\end{array}$} & \multicolumn{2}{|c|}{ Observations } \\
\hline GPKMP & overall & & & & & $\mathrm{N}=$ & 55 \\
\hline & between & & 43.29004 & 259.5455 & 371.3636 & $\mathrm{n}=$ & 5 \\
\hline & within & & 36.756 & 226.9455 & 365.9455 & $\mathrm{~T}=$ & 11 \\
\hline \multirow[t]{3}{*}{ NENTNC } & overall & 18.13455 & 3.92802 & 10.3 & 26.8 & $\mathrm{~N}=$ & 55 \\
\hline & between & & 3.910821 & 13.17273 & 23.6 & $\mathrm{n}=$ & 5 \\
\hline & within & & 1.722538 & 12.93455 & 21.80727 & $\mathrm{~T}=$ & 11 \\
\hline \multirow[t]{3}{*}{ CSP } & overall & $1.75 E+08$ & $1.64 \mathrm{E}+08$ & 688469 & $6.58 \mathrm{E}+08$ & $\mathrm{~N}=$ & 55 \\
\hline & between & & $1.54 \mathrm{E}+08$ & 8052653 & $4.07 \mathrm{E}+08$ & $\mathrm{n}=$ & 5 \\
\hline & within & & $8.68 E+07$ & $-3.07 E+07$ & $4.26 \mathrm{E}+08$ & $\mathrm{~T}=$ & 11 \\
\hline
\end{tabular}

Información procesada en Stata.

Los resultados de la estimación se muestran en la tabla 8. Los distintos métodos de Datos de Panel utilizados muestran significancia estadística, a excepción de CSP de datos agrupados. Para todos los casos existe una 
relación positiva entre ingresos por CSP sobre el gasto per cápita del $40 \%$ de la población más pobre. Sin embargo, la magnitud de este efecto positivo es exigua. El efecto tendencial muestra un incremento promedio similar de 11 puntos anuales (equivalente a 0.91 céntimos por mes) en todas las regiones a excepción del panel dinámico cuya significación y magnitud es menor en comparación con sus pares. Una virtud del modelo longitudinal es su capacidad de capturar los efectos individuales que pueden estar presentes y por definición, invariantes en el tiempo. Los resultados en el modelo de variables dicotómicas de efecto fijos muestran que los efectos no observables inciden negativamente en el gasto per cápita de la población más pobre. Los efectos heterogéneos por región muestran que Loreto es la región con menor contribución al gasto per cápita del $40 \%$ de la población más pobre ${ }^{11}$, mientras que Huánuco y Piura tienen efectos negativos, pero en menor proporción.

Tabla 8

Resultados de la estimación de Datos de Panel, 2007 - 2017

Hipótesis 2

\begin{tabular}{|c|c|c|c|c|}
\hline GPKMP & Datos Agrupados & $\begin{array}{c}\text { Efectos Fijos con } \\
\text { Variable Dicotómica }\end{array}$ & $\begin{array}{c}\text { Efectos } \\
\text { Aleatorios }\end{array}$ & $\begin{array}{l}\text { Panel Dinámico } \\
\text { (Arellano y Bond) }\end{array}$ \\
\hline \multirow[t]{2}{*}{ GPKMP $_{-1}$} & & & & $0.5063814^{* *}$ \\
\hline & & & & $(0.1730842)$ \\
\hline \multirow[t]{2}{*}{ CSP } & $1.21 \mathrm{E}-08$ & $6.615 \mathrm{e}-08^{* * *}$ & $6.525 \mathrm{e}-08^{* * *}$ & $4.02 \mathrm{e}-08^{*}$ \\
\hline & $(0.0000000347)$ & $(0.000000018)$ & $(0.0000000177)$ & $(0.00000001 .83)$ \\
\hline \multirow[t]{2}{*}{$\mathrm{t}$} & $10.914548^{* * *}$ & $11.109102^{* * *}$ & $11.10585^{* * *}$ & $4.634226^{*}$ \\
\hline & (1.780338) & (0.489179) & $(0.4853936)$ & (2.173024) \\
\hline \multirow[t]{2}{*}{ Dummy Ucayali } & & $69.317553^{* * *}$ & & \\
\hline & & (5.505031) & & \\
\hline \multirow[t]{2}{*}{ Dummy Piura } & & $38.709381^{* * *}$ & & \\
\hline & & (5.751581) & & \\
\hline \multirow[t]{2}{*}{ Dummy Tumbes } & & $118.5374^{* * *}$ & & \\
\hline & & (5.18131) & & \\
\hline \multirow[t]{2}{*}{ Dummy Huánuco } & & $31.744893^{* * *}$ & & \\
\hline & & $(6.338164)$ & & \\
\hline \multirow[t]{2}{*}{ CONST } & $-21654.971^{* * *}$ & $-22107.518^{* * *}$ & $-22049.155^{* * *}$ & \\
\hline & (3582.477) & $(984.8024)$ & (977.2762) & \\
\hline r2 & 0.41958 & 0.96010 & 0.39570 & \\
\hline $\mathrm{N}$ & 55 & 55 & 55 & 45 \\
\hline
\end{tabular}

legend: $\quad * \mathrm{p}<0.05$; $^{* *} \mathrm{p}<0.01$; $^{* *} \mathrm{p}<0.001$

( ) errores estándar

Elaborado por el autor. Stata. 
En relación a la post estimación, la autocorrelación medida a través del test de Pasaran indica una probabilidad de 0.3417, por lo que se descarta autocorrelación y se acepta independencia cruzada de los residuos. El test cross sectional dependence: Breusch Pagan LM muestra un Pr de 0.3086, confirmando el test Pesaran. El test de autocorrelación serial de Wooldridge para panel de datos el cual determina que los residuos de la serie de cada sujeto de manera individual están correlacionados señala un valor $\mathrm{p}>0.05$, lo que se descarta autocorrelación serial. La probabilidad chi cuadrada muestra un valor $\mathrm{p}>0.05$, por lo que se descarta presencia de heteroscedasticidad.

En relación a la estimación dinámica, el test de Sargan ${ }^{12}$ el cual descarta sobre identificación y validez de instrumentos, señala un valor Prob>chi2 de 0.1856, por tanto, los instrumentos utilizados para la estimación son correctos. Con respecto a la autocorrelación el estadístico de segundo orden $\operatorname{Ar}(2)$ muestra un valor Pr>z 0.8849 por lo que no se rechaza la hipótesis nula de No Autocorrelación.

Con respecto a la estimación del efecto del CSP sobre el porcentaje de jóvenes entre 15 y 24 que no estudian, no trabajan y no reciben capacitación, los resultados se muestran en la tabla 9. Las estimaciones evidencian que sólo los datos agrupados de panel muestran significancia estadística con magnitudes muy pequeñas en relación al efecto del CSP. El efecto positivo indica que los ingresos por CSP aumentó la proporción sobre trabajo decente de jóvenes entre 18 y 24 años. En relación a los efectos no observados utilizando variables dicotómicas de efectos fijos por región, Loreto muestra mayor explicación de efectos no observables, seguido de Tumbes, Piura, Ucayali y Huánuco.

La prueba de diagnóstico muestra ausencia de autocorrelación. El test de Pesaran muestra un valor pr 0.1511 y el test de Breusch Pagan LM muestra un Pr de 0.5151. El modelo muestra ausencia de heteroscedasticidad con un p value de 0.0961. El test de autocorrelación serial de Wooldridge el cual determina que los residuos de la serie de cada sujeto de manera individual están correlacionados señala un valor Prob > $\mathrm{F}=0.1333$ mayor a 0.05 , lo que se descarta autocorrelación serial. Con relación al modelo dinámico, el test de Sargan, descarta sobre identificación y validez de instrumentos señala un Prob $>$ chi2 $=0.1727$. El 
test de autocorrelación el estadístico de segundo orden $\operatorname{Ar}(2)$ muestra un valor $\operatorname{Pr}>\mathrm{z}=0.4471$ por lo que se rechaza la hipótesis nula de No Autocorrelación.

\section{Tabla 9}

Resultados de la estimación de Datos de Panel, 2007 - 2017

Hipótesis 3

\begin{tabular}{|c|c|c|c|c|}
\hline NENONC & Datos Agrupados & $\begin{array}{l}\text { Efectos Fijos con } \\
\text { Variable Dicotómica }\end{array}$ & Efectos Aleatorios & $\begin{array}{l}\text { Panel Dinámico } \\
\text { (Arellano y Bond) }\end{array}$ \\
\hline \multirow[t]{2}{*}{ NENONC $_{-1}$} & & & & 0.12386058 \\
\hline & & & & (.1664509) \\
\hline \multirow[t]{2}{*}{ CSP } & $1.122 \mathrm{e}-08^{* * *}$ & $-5.19 \mathrm{E}-10$ & 4.37E-10 & $-2.05 \mathrm{E}-09$ \\
\hline & $(2.95 \mathrm{e}-09)$ & $(2.89 \mathrm{e}-09)$ & ( $2.82 \mathrm{e}-09)$ & ( $2.96 \mathrm{e}-09)$ \\
\hline \multirow[t]{2}{*}{ t } & .02168422 & -.02059452 & -.0171556 & -.06197351 \\
\hline & (.1513574) & $(.0785224)$ & $(.0791323)$ & .0882121 \\
\hline \multirow[t]{2}{*}{ Dummy Ucayali } & & $-7.8024522^{* * *}$ & & \\
\hline & & $(.8836607)$ & & \\
\hline \multirow[t]{2}{*}{ Dummy Piura } & & $-4.6107784^{* * *}$ & & \\
\hline & & $(.9232366)$ & & \\
\hline \multirow[t]{2}{*}{ Dummy Tumbes } & & $-4.5254003^{* * *}$ & & \\
\hline & & $(.8316974)$ & & \\
\hline \multirow[t]{2}{*}{ Dummy Huánuco } & & $-10.544998^{* * *}$ & & \\
\hline & & (1.017394) & & \\
\hline \multirow[t]{2}{*}{ CONST } & -27.455571 & 65.158083 & 52.57531 & \\
\hline & (304.5682) & (158.0793) & (159.2865) & \\
\hline r2 & 0.21750259 & & & \\
\hline $\mathrm{N}$ & 55 & 55 & 55 & 45 \\
\hline
\end{tabular}

legend: ${ }^{*} \mathrm{p}<0.05 ;{ }^{* *} \mathrm{p}<0.01 ;{ }^{* * *} \mathrm{p}<0.001$

() errores estándar

Elaborado por el autor.

\section{Discusión}

Centramos una primera observación que consideramos oportuno precisar desde el punto de vista estadístico del modelo VAR. El modelo es afectado por la endogeneidad por lo que no se acostumbra analizar los coeficientes ni su significancia estadística, como tampoco no es sugerible excluirse tales variables por su significancia estadística. Las razones radican en que las estimaciones ecuación por ecuación por mínimos cuadrados ordinarios (MCO) es eficiente y, por otro, que ciertamente la colinealidad inherente del 
modelo da paso a reducir los valores estadísticos t. Por tanto, ésta no se debe a la falta de contenido informativo de las variables. Por otro lado, el modelo permite calcular con bastante precisión el ajuste del modelo $\left(\mathrm{R}^{2}\right)$, así como la prueba de significancia individual (t) y conjunta (F). Sin embargo, tal como señala Novales (2017), "no cabe hacer interpretaciones de coeficientes individuales en distintos retardos, ni llevar a cabo contrastes de hipótesis sobre coeficientes individuales" (p. 8).

Por lo señalado, las interpretaciones en el presente estudio se enfocaron en la dirección de los coeficientes y alternativamente, en la magnitud. Tomando relevancia para el análisis las funciones impulso respuesta y descomposición de varianzas.

El efecto del ingreso por CSP en la actividad económica regional (PBI) y la productividad en Loreto muestra escaso impacto, tanto desde la magnitud, como del impulso respuesta hacia las variables de interés. Una sugerente explicación se debe a la cantidad presupuestal destinada a proyectos de inversión que permitan invertir en infraestructura básica de desarrollo como escuelas, centros de salud, carreteras, centros de producción, etc. Al analizar para el periodo 2007 - 2018 el destino del gasto ${ }^{13}$ se encuentra que en promedio el gasto destinado a inversiones y/o activos no financieros dista a lo señalado por Ley $(80 \%$ en gastos de inversión y $20 \%$ en gastos corriente - destinado a mantenimiento de infraestructura social). Más aun considerando la calidad del gasto y la insuficiente y mediocre ejecución presupuestal de la inversión pública (puesta en marcha de las ejecutadas), lo que escapa al objetivo del presente estudio. La figura 8 muestra lo indicado.

En relación al análisis de la contribución del ingreso por CSP a las variables de reducción de desigualdad (ingreso del 40\% de más bajos ingresos) y trabajo decente de jóvenes entre 15 y 24 años para las cinco regiones de estudio, los resultados aplicando los distintos datos de panel muestran magnitudes extremadamente bajo para el primero y desfavorable para el segundo. Al respecto, la trayectoria histórica y lo analizado en este estudio conduce a enfocarse en los efectos negativos. Las distintas regiones de estudio han recibido destacados ingresos por la explotación de hidrocarburos. Sin embargo, los niveles de contribución a los aspectos socioeconómicos (producción, productividad, desigualdad, empleo) han 
sido mínimas. La tabla 10 muestra los ingresos percibidos en 2007 - 2017, por las cinco regiones de estudio.

\section{Figura 8}

Ejecución del gasto en inversiones. Gobierno Regional de Loreto. Periodo 2007 - 2018

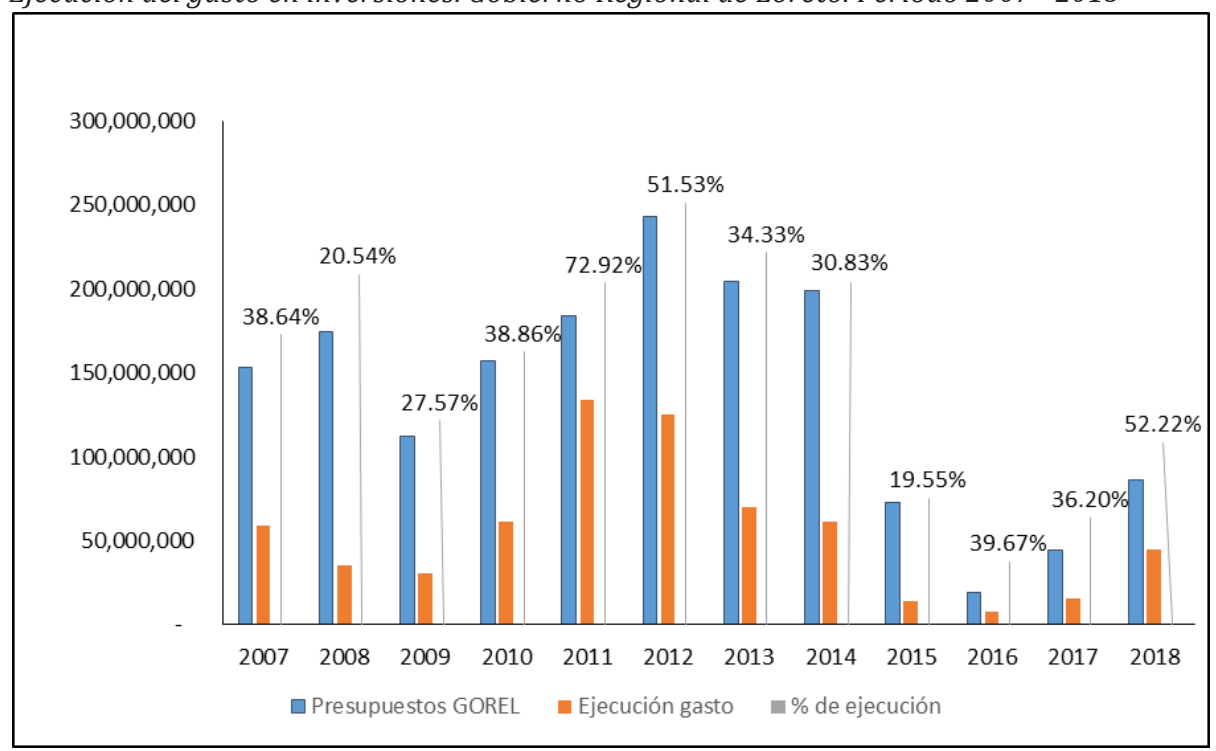

Elaboración propia

Tabla 10

Ingresos por Canon y Sobrecanon Petrolero. Periodo 2007 - 2017 (Soles)

\begin{tabular}{cccccr}
\hline Año & Loreto & Ucayali & Piura & Tumbes & Huánuco \\
\hline 2007 & $295,037,083.47$ & $107,846,342.84$ & $281,066,473.57$ & $71,693,562.80$ & $762,236.08$ \\
2008 & $334,851,083.57$ & $123,175,603.25$ & $392,292,499.98$ & $123,459,115.95$ & $1,141,119.81$ \\
2009 & $177,569,011.23$ & $74,510,586.58$ & $270,748,822.40$ & $85,190,467.75$ & $688,469.42$ \\
2010 & $223,130,550.52$ & $84,509,991.26$ & $337,117,840.41$ & $116,252,947.71$ & $1,041,041.12$ \\
2011 & $281,068,358.87$ & $98,415,941.01$ & $454,114,302.62$ & $152,186,038.23$ & $1,534,630.13$ \\
2012 & $377,872,824.76$ & $144,088,719.73$ & $658,130,492.28$ & $214,750,054.90$ & $1,534,630.13$ \\
2013 & $344,009,976.73$ & $122,455,510.47$ & $604,553,466.29$ & $191,838,392.83$ & $2,040,700.97$ \\
2014 & $335,847,190.20$ & $119,035,654.81$ & $592,933,408.82$ & $218,713,319.94$ & $10,133,413.95$ \\
2015 & $119,886,811.38$ & $60,342,462.94$ & $321,581,550.03$ & $112,109,808.28$ & $25,953,909.38$ \\
2016 & $29,588,171.31$ & $28,357,763.27$ & $263,460,484.12$ & $86,170,532.05$ & $15,096,488.47$ \\
2017 & $66,842,597.71$ & $28,296,807.86$ & $302,220,615.97$ & $96,063,345.66$ & $28,652,545.22$ \\
TOTAL & $2,585,703,659.75$ & $991,035,384.02$ & $4,478,219,956.49$ & $1,468,427,586.10$ & $88,579,184.68$ \\
\hline
\end{tabular}

Fuente. Estadísticas hidrocarburos PERUPETRO 
Un conocimiento a priori, permite concluir que el despilfarro, la corrupción, la falta de control, la calidad de los proyectos de desarrollo, entre otros, son los determinantes de la falta de respuesta impulso al desarrollo de las regiones que muestran bajos índices económicos y sociales. La pandemia mundial del COVID 19 desatado a inicios del 2020 ha puesto en evidencia la fragilidad de la infraestructura económica, social, institucional y de gobernanza política en los distintos niveles de gestión pública en Perú.

Con respecto al único estudio empírico realizado en el Perú por Correa y Morocho (2016) del impacto del CSP en la actividad económica y social en la región Piura, los resultados son similares parcialmente, a los hallazgos del presente estudio. Los efectos en la actividad económica en ambos estudios fueron positivos y no significativos. La función impulso respuesta reacciona de manera similar en ambos estudios.

Una observación entre ambos estudios radica en que el impacto del CSP en el caso del estudio aplicada a la región Piura muestra resultados diferenciados: La contribución del CSP del gobierno regional es negativa mientras que la del gobierno local es positiva. El estudio aquí encontrado encontró resultados inversos. El efecto del CSP del gobierno regional es positivo y negativo para el gobierno local, aunque estadísticamente no significativos. En relación a la función impulso respuesta, para el caso de la región Piura la actividad económica responde a choques positivos por la inversión del gobierno local y nula para el gobierno regional. Los resultados en la región Loreto muestran que los choques del gobierno regional son positivos, mientras que la del gobierno local es negativa.

En base a los resultados encontrados aceptamos la hipótesis 1 y 2 observando magnitudes e impacto extremadamente exiguo sobre las variables de interés y descartar la hipótesis 3. Por tanto, podemos afirmar como un conocimiento a priori y demostración empírica a posteriori sobre los efectos entre las variables de estudio que parece ser que la maldición de los recursos sostenidos en el acápite teórico no está en los efectos negativos sobre el crecimiento, sino en la dependencia y probable mal uso que se le da a los recursos determinados por el despilfarro, la ineficiencia del capital humano, la pereza institucional, corrupción 
y la falta de un marco legal más rígido para el control de los recursos (trampa de la grapa).

\section{Conclusiones}

El estudio buscó encontrar dos efectos: Primero, el efecto de los ingresos por CSP sobre el PBI y la productividad en la región Loreto. Segundo, el efecto del CSP sobre dos variables socioeconómicas en cinco regiones, Loreto, Piura, Tumbes, Huánuco y Ucayali: el gasto per cápita entre al 40\% de la población más pobre, y sobre el porcentaje de jóvenes entre 15 y 24 que no estudian, no trabajan y no reciben capacitación.

Las estimaciones del primer modelo aceptan la hipótesis nula que los ingresos tuvieron efecto sobre el PBI y la productividad en la región Loreto. Al respecto, se destacan dos aspectos importantes sobre estos resultados. Primero, el resultado es negativo para el periodo $1994-2012$, siendo positivo para el periodo 2013 - 2019. Los resultados son disimiles en cuanto a que un aumento en el CSP tiene efectos negativos y una disminución tiene efectos positivos sobre el PBI y la productividad regional, puede estar xde la distribución normal de los residuos. No obstante a lo señalado, el impulso del CSP sobre el PBI y la productividad regional es insignificante debido a la magnitud mostrada.

En relación al efecto desagregado, el CSP asignado al gobierno regional tuvieron efecto positivo y el CSP asignado a los gobiernos locales mostraron efecto negativo, en ambos casos las magnitudes no son significativas. El impulso del CSP regional representa un 11\%, mientras que el CSP de los gobiernos locales es exigua.

Los resultados para comprobar la hipótesis 2 (2007-2017) el cual sostiene que el CSP sobre el gasto per cápita del 40\% de la población más pobre por región es positivo y estadísticamente significativo, se acepta. Sin embargo, la magnitud es extremadamente pequeña. Los efectos individuales no observados en todos los casos son negativos.

La hipótesis 3 (2007-2017) el cual sostiene que el ingreso por CSP tuvo efecto en la disminución del porcentaje de jóvenes entre 15 y 24 que no estudian, no trabajan y no reciben capacitación, ésta se rechaza. No existió efecto favorable. 


\section{Referencias Bibliograficas}

Clancy, C y Kerremans, S (2016). La dependencia petrolera y la Amazonia Peruana. Instituto Chaikuni. Disponible en: http://extractivismo.com/wpcontent/uploads/2016/07/DependenciaPetroleraAmazoniaPeru.pdf

Correa, H y Morocho, J (2016). Análisis del impacto económico y social del canon y sobre canon petrolero en la región Piura: período 1984-2014. CIES. Disponible en: Análisis del impacto económico y social del canon y sobre canon petrolero en la región Piura: período 1984-2014

Domínguez, R (2009). Staple theory y crecimiento liderado por las exportaciones. México en el contexto norte y latinoamericano. Disponible en: https://www. academia.edu/2356618/_Staple_theory_y_crecimiento_liderado_por_las_ exportaciones._M\%C3\%A9xico_en_el_contexto_norte_y_latinoamericano_en_M.E._Romero_y_A._Carrillo_coords._Empresa_y_agricultura_comercial_en_el_Noroeste_de_M\%C3\%A9xico._Historia_econ\%C3\%B3mica_y_ tendencias_actuales._UNAM_M\%C3\%A9xico_2009_p\%C3\%A1gs._421-457

Gaviria, M (2010). Apuntes de economía regional. Universidad Católica Popular del Risaralda. http://biblioteca.utec.edu.sv/siab/virtual/elibros_internet/55767.pdf

Instituto Nacional de Estadística e Informática (2018). Línea de base de los principales indicadores disponibles de los objetivos de desarrollo sostenible (ODS). https://www.inei.gob.pe/media/MenuRecursivo/publicaciones_digitales/Est/Lib1578/libro.pdf

Labarthe, R (22 de abril del 2019). 43\% del canon petrolero no se destinó a proyectos. Diario La República. En: https://larepublica.pe/economia/145509743-canon-petrolero-destino-proyectos/

Lanteri, L (2016). Efectos de la enfermedad holandesa ('Dutch disease') Alguna evidencia para Argentina. Revista de Economía del Rosario. Vol. $18 \mathrm{~N}^{\circ} 1$. Doi: dx.doi.org/10.12804/rev.econ.rosario.18.02.2015.02

López, M (2018). El canon petrolero como fuente de financiamiento del gasto en la región Loreto. Colegio de Economistas de Loreto. Recuperado de: https:// www.celor.org/articulo-n-23

Ministerio de Energía y Minas (2010). Canon y Sobrecanon petrolero. Importancia del canon petrolero para Piura, Tumbes, Loreto, Ucayali y Huánuco proyecciones 2010. Disponible en: http://minem.gob.pe

El Peruano (20 de setiembre del 2018). Normas legales. https://busquedas.elperuano.pe/download/url/ley-que-modifica-la-ley-27506-ley-de-canon-a-finde-promov-ley-n-30848-1693569-1 
Novales, A (2017). Modelo vectoriales autorregresivos (VAR). Universidad Complutense. Disponible en: https://www.ucm.es/data/cont/media/www/pag41459/VAR.pdf

Orihuela, C (2014). Evaluando el desarrollo sostenible en la región Loreto: una aplicación de un enfoque basado en sostenibilidad débil. CIES. Disponible en: http://cies.org.pe/sites/default/files/investigaciones/informe_final_3. pdf

Perry, G y Olivera M (2009). El impacto del petróleo y la minería en el desarrollo regional y local en Colombia. CAF N ${ }^{\circ}$ 2009/06. Disponible en: http://data. infraestructuravisible.org/documents/Papers/Perry_Olivera_2009.pdf

Poinsot, P y Ruault, J (2019). Economic-base theory and highly-open economies: incorporating day-to- day mobility. HAL Id: hal-02269336. Disponible en: https://hal.archives-ouvertes.fr/hal-02269336

Ravago, M y Roumasset, J (2015). Elección pública y la maldición generalizada de recursos. https://www.sciencedirect.com/topics/social-sciences/dutchdisease

Reina, L. Alejo, D y Devia N (2018). Análisis regional de Colombia y su maldición de recursos naturales: Cambios institucionales tardíos. Disponible en: http://dx.doi.org/10.4067/S0250-71612018000100125

Sachs, J y Warner, A (1995). Natural resource abundance and economic grotwh. NBER working paper series 5398. https://www.nber.org/papers/w5398. pdf

Sociedad Peruana de Hidrocarburos (2013). Impacto del Sector Hidrocarburos en la Economía del Perú. En: http://www.enernews.com/media/briefs/impacto-del-sector-hidrocarburos_1177.pdf

Statista (2020). Evolución anual del precio medio del petróleo crudo fijado por la Organización de Países Exportadores de Petróleo (OPEP) de 1960 a 2019. Recuperado de https://es.statista.com/estadisticas/635114/precio-mediodel-crudo-fijado-por-la-opep/

Tello, M (2006). Las teorías del desarrollo económico local y la teoría y práctica del proceso de descentralización en los países en desarrollo. Documento de trabajo 247. PUCP. Recuperado de http://files.pucp.edu.pe/departamento/ economia/DDD247.pdf 
ANEXOS

\section{Anexo 1}

Test de Dickey Fuller

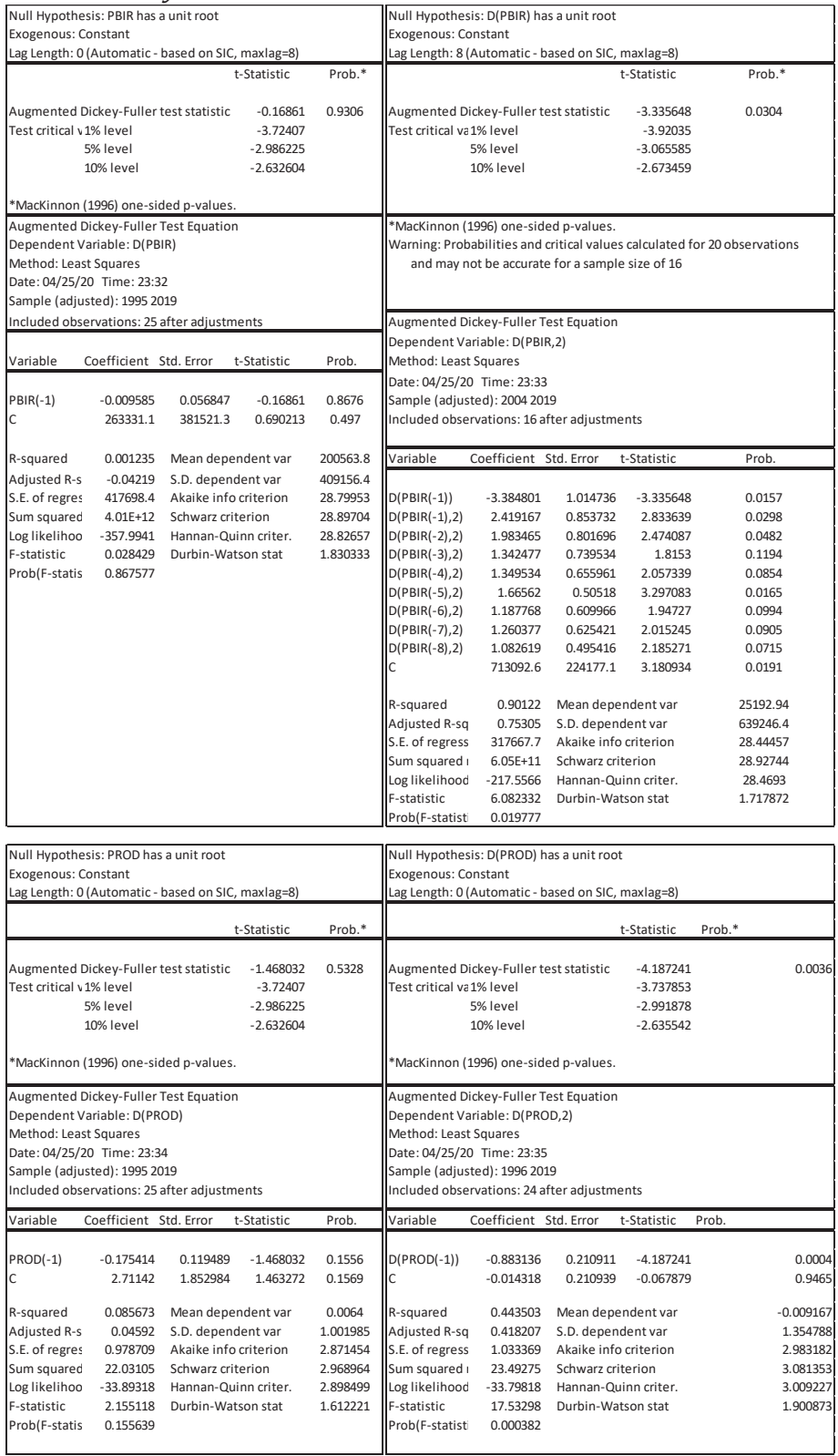


Pensamiento Crítico Vol. 26. ํํㄹ 2

Anexo 1. Continuación...

\begin{tabular}{|c|c|c|c|c|c|c|c|c|c|}
\hline \multicolumn{5}{|c|}{$\begin{array}{l}\text { Null Hypothesis: CANON has a unit root } \\
\text { Exogenous: Constant } \\
\text { Lag Length: } 0 \text { (Automatic - based on SIC, maxlag=8) }\end{array}$} & \multicolumn{5}{|c|}{$\begin{array}{l}\text { Null Hypothesis: } \mathrm{D}(\mathrm{CANON}) \text { has a unit root } \\
\text { Exogenous: Constant } \\
\text { Lag Length: } 0 \text { (Automatic - based on SIC, maxlag=8) }\end{array}$} \\
\hline \multicolumn{4}{|r|}{ t-Statistic } & Prob.* & \multicolumn{4}{|r|}{ t-Statistic } & Prob.* \\
\hline \multirow{2}{*}{\multicolumn{4}{|c|}{$\begin{array}{l}\text { Augmented Dickey-Fuller test statistic } \\
\text { Test critical v } 1 \% \text { level } \\
5 \% \text { level } \\
10 \% \text { level } \\
\text { *MacKinnon (1996) one-sided p-values. }\end{array}$}} & 0.2889 & \multirow{2}{*}{\multicolumn{4}{|c|}{\begin{tabular}{|} 
Augmented Dickey-Fuller test statistic \\
Test critical vã $1 \%$ level \\
$5 \%$ level \\
$10 \%$ level \\
*MacKinnon (1996) one-sided p-values.
\end{tabular}}} & 0.0039 \\
\hline & & & & & & & & & \\
\hline \multicolumn{5}{|c|}{$\begin{array}{l}\text { Augmented Dickey-Fuller Test Equation } \\
\text { Dependent Variable: D(CANON) } \\
\text { Method: Least Squares } \\
\text { Date: } 04 / 25 / 20 \text { Time: } 23: 35 \\
\text { Sample (adjusted): } 19952019 \\
\text { Included observations: } 25 \text { after adjustments }\end{array}$} & \multicolumn{5}{|c|}{$\begin{array}{l}\text { Augmented Dickey-Fuller Test Equation } \\
\text { Dependent Variable: D(CANON,2) } \\
\text { Method: Least Squares } \\
\text { Date: 04/25/20 Time: } 23: 36 \\
\text { Sample (adjusted): } 19962019 \\
\text { Included observations: } 24 \text { after adjustments }\end{array}$} \\
\hline Variable & Coefficient & Std. Error & t-Statistic & Prob. & Variable & Coefficient & Std. Error & t-Statistic & Prob. \\
\hline CANON(-1) & -0.269036 & 0.135175 & -1.990283 & 0.0586 & D(CANON $(-1)$ & -0.87904 & 0.211687 & -4.152545 & 0.0004 \\
\hline C & 53374473 & 28711939 & 1.858964 & 0.0759 & C & 2452314 & 15050849 & 0.162935 & 0.8721 \\
\hline R-squared & 0.146923 & \multicolumn{2}{|c|}{ Mean dependent var } & 2846717 & R-squared & 0.4394 & \multicolumn{2}{|c|}{ Mean dependent var } & 235378.6 \\
\hline Adjusted R-s & 0.109833 & \multicolumn{2}{|c|}{ S.D. dependent var } & 71073318 & Adjusted R-sq & 0.413918 & \multicolumn{2}{|c|}{ S.D. dependent var } & 96252923 \\
\hline S.E. of regres & 67056734 & \multicolumn{2}{|c|}{ Akaike info criterion } & 38.95659 & S.E. of regress & 73687401 & \multicolumn{2}{|c|}{ Akaike info criterion } & 39.14822 \\
\hline Sum squared & $1.03 \mathrm{E}+17$ & \multicolumn{2}{|c|}{ Schwarz criterion } & 39.0541 & Sum squared & 1.19E+17 & \multicolumn{2}{|c|}{ Schwarz criterion } & 39.24639 \\
\hline Log likelihoo & -484.9574 & \multirow{2}{*}{\multicolumn{2}{|c|}{$\begin{array}{l}\text { Hannan-Quinn criter. } \\
\text { Durbin-Watson stat }\end{array}$}} & 38.98364 & Log likelihooc & -467.7786 & \multirow{2}{*}{\multicolumn{2}{|c|}{$\begin{array}{l}\text { Hannan-Quinn criter. } \\
\text { Durbin-Watson stat }\end{array}$}} & 39.17426 \\
\hline F-statistic & 3.961225 & & & 1.591197 & F-statistic & 17.24363 & & & 1.958083 \\
\hline Prob(F-statis & 0.058574 & & & & Prob(F-statist & 0.000416 & & & \\
\hline
\end{tabular}

\begin{tabular}{|c|c|c|c|c|c|c|c|c|c|}
\hline \multicolumn{5}{|c|}{$\begin{array}{l}\text { Null Hypothesis: PP has a unit root } \\
\text { Exogenous: Constant } \\
\text { Lag Length: } 0 \text { (Automatic - based on SIC, maxlag=8) }\end{array}$} & \multicolumn{5}{|c|}{$\begin{array}{l}\text { Null Hypothesis: D(PP) has a unit root } \\
\text { Exogenous: Constant } \\
\text { Lag Length: } 0 \text { (Automatic - based on SIC, maxlag=8) }\end{array}$} \\
\hline & & & t-Statistic & Prob.* & & & & t-Statistic & Prob.* \\
\hline \multirow{2}{*}{\multicolumn{4}{|c|}{$\begin{array}{l}\text { Augmented Dickey-Fuller test statistic } \\
\text { Test critical v } 1 \% \text { level } \\
5 \% \text { level } \\
10 \% \text { level } \\
\text { *MacKinnon (1996) one-sided p-values. }\end{array}$}} & 0.4898 & \multirow{2}{*}{\multicolumn{3}{|c|}{\begin{tabular}{|} 
Augmented Dickey-Fuller test statistic \\
Test critical vã $1 \%$ level \\
$5 \%$ level \\
$10 \%$ level \\
*MacKinnon (1996) one-sided p-values.
\end{tabular}}} & $\begin{array}{l}-4.094724 \\
-3.737853 \\
-2.991878 \\
-2.635542\end{array}$ & 0.0044 \\
\hline & & & & & & & & & \\
\hline $\begin{array}{l}\text { Augmented D } \\
\text { Dependent V } \\
\text { Method: Leas } \\
\text { Date: } 04 / 25 / 2 \\
\text { Sample (adju } \\
\text { Included obse }\end{array}$ & $\begin{array}{l}\text { Dickey-Fuller } \\
\text { /ariable: D(PP } \\
\text { st Squares } \\
20 \text { Time: } 23: 3 \\
\text { usted): } 19952 \\
\text { servations: } 25\end{array}$ & $\begin{array}{l}\text { Pest Equatior } \\
\text { P) } \\
36 \\
2019 \\
\text { after adjustm }\end{array}$ & nents & & \multicolumn{5}{|c|}{$\begin{array}{l}\text { Augmented Dickey-Fuller Test Equation } \\
\text { Dependent Variable: } \mathrm{D}(\mathrm{PP}, 2) \\
\text { Method: Least Squares } \\
\text { Date: } 04 / 25 / 20 \text { Time: } 23: 37 \\
\text { Sample (adjusted): } 19962019 \\
\text { Included observations: } 24 \text { after adjustments }\end{array}$} \\
\hline Variable & Coefficient & Std. Error & t-Statistic & Prob. & Variable & Coefficient & Std. Error & t-Statistic & Prob. \\
\hline $\mathrm{PP}(-1)$ & -0.165001 & 0.106088 & -1.555326 & 0.1335 & $D(P P(-1))$ & -0.867443 & 0.211844 & -4.094724 & 0.0005 \\
\hline C & 10.40314 & 6.369063 & 1.633387 & 0.116 & C & 1.744532 & 3.715765 & 0.469495 & 0.6433 \\
\hline R-squared & 0.095166 & \multicolumn{2}{|c|}{ Mean dependent var } & 2.0156 & R-squared & 0.432504 & \multicolumn{2}{|c|}{ Mean dependent var } & -0.21625 \\
\hline Adjusted R-s & 0.055826 & \multicolumn{2}{|c|}{ S.D. dependent var } & 17.43685 & Adjusted R-sq & 0.406708 & \multicolumn{2}{|c|}{ S.D. dependent var } & 23.43598 \\
\hline S.E. of regres & 16.94315 & \multicolumn{2}{|c|}{ Akaike info criterion } & 8.574223 & S.E. of regress & 18.05166 & \multicolumn{2}{|c|}{ Akaike info criterion } & 8.704008 \\
\hline Sum squared & 6602.617 & \multicolumn{2}{|c|}{ Schwarz criterion } & 8.671733 & Sum squared & 7168.974 & \multicolumn{2}{|c|}{ Schwarz criterion } & 8.802179 \\
\hline Log likelihoo & -105.1778 & \multicolumn{2}{|c|}{ Hannan-Quinn criter. } & 8.601268 & Log likelihooc & -102.4481 & \multirow{2}{*}{\multicolumn{2}{|c|}{$\begin{array}{l}\text { Hannan-Quinn criter. } \\
\text { Durbin-Watson stat }\end{array}$}} & 8.730053 \\
\hline F-statistic & 2.41904 & \multicolumn{2}{|c|}{ Durbin-Watson stat } & 1.6285 & F-statistic & 16.76676 & & & 1.913509 \\
\hline Prob(F-statis & 0.133522 & & & & Prob(F-statist & 0.000479 & \multicolumn{2}{|c|}{ Durbin-watson stat } & \\
\hline
\end{tabular}


Anexo 2

Criterio de selección de rezagos

\begin{tabular}{|c|c|c|c|c|c|c|}
\hline \multicolumn{7}{|c|}{ VAR Lag Order Selection Criteria } \\
\hline \multicolumn{7}{|c|}{ Endogenous variables: DPBIR DPROD DCANON } \\
\hline \multicolumn{7}{|c|}{ Exogenous variables: C D1 } \\
\hline \multicolumn{7}{|c|}{ Date: $04 / 26 / 20$ Time: $17: 22$} \\
\hline \multicolumn{7}{|c|}{ Sample: 19942019} \\
\hline \multicolumn{7}{|c|}{ Included observations: 21} \\
\hline Lag & $\log L$ & LR & FPE & AIC & SC & HQ \\
\hline 0 & -724.0894 & NA* & $3.17 \mathrm{e}+26^{*}$ & $69.53232^{*}$ & $69.83076^{*}$ & $69.59709 *$ \\
\hline 1 & -716.1738 & 12.06187 & $3.61 \mathrm{E}+26$ & 69.6356 & 70.38169 & 69.79752 \\
\hline 2 & -707.1478 & 11.17504 & $3.95 \mathrm{E}+26$ & 69.63312 & 70.82686 & 69.89219 \\
\hline 3 & -701.4213 & 5.453798 & $6.76 \mathrm{E}+26$ & 69.94489 & 71.58628 & 70.30111 \\
\hline 4 & -691.191 & 6.820165 & $9.73 \mathrm{E}+26$ & 69.82772 & 71.91676 & 70.2811 \\
\hline \multicolumn{7}{|c|}{ * indicates lag order selected by the criterion } \\
\hline \multicolumn{7}{|c|}{ LR: sequential modified LR test statistic (each test at $5 \%$ level) } \\
\hline \multicolumn{7}{|c|}{ FPE: Final prediction error } \\
\hline \multicolumn{7}{|c|}{ AIC: Akaike information criterion } \\
\hline \multicolumn{7}{|c|}{ SC: Schwarz information criterion } \\
\hline HQ: Hannz & nformation & ion & & & & \\
\hline
\end{tabular}

\section{Anexo 3}

Descomposición de Varianza. Modelo VAR 1

\begin{tabular}{|crrrr|}
\hline \multicolumn{5}{|c|}{ Variance Decomposition of DPBIR: } \\
\hline Period & S.E. & DPBIR & DPROD & DCANON \\
\hline 1 & 429430.5 & 100 & 0 & 0 \\
2 & 452121.3 & 90.46163 & 0.061219 & 9.477153 \\
3 & 454142.7 & 90.07522 & 0.475840 & 9.448940 \\
4 & 454326.4 & 90.03562 & 0.484725 & 9.479651 \\
5 & 454340.4 & 90.0343 & 0.486622 & 9.479076 \\
6 & 454340.7 & 90.03426 & 0.486626 & 9.479112 \\
7 & 454340.8 & 90.03426 & 0.486628 & 9.479114 \\
8 & 454340.8 & 90.03426 & 0.486628 & 9.479114 \\
9 & 454340.8 & 90.03426 & 0.486628 & 9.479114 \\
10 & 454340.8 & 90.03426 & 0.486628 & 9.479114 \\
11 & 454340.8 & 90.03426 & 0.486628 & 9.479114 \\
12 & 454340.8 & 90.03426 & 0.486628 & 9.479114 \\
13 & 454340.8 & 90.03426 & 0.486628 & 9.479114 \\
14 & 454340.8 & 90.03426 & 0.486628 & 9.479114 \\
15 & 454340.8 & 90.03426 & 0.486628 & 9.479114 \\
\hline
\end{tabular}

\begin{tabular}{|crrrr|}
\hline \multicolumn{5}{|c|}{ Variance Decomposition of DPROD: } \\
\hline Period & S.E. & DPBIR & DPROD & DCANON \\
\hline 1 & 1.10736 & 82.55518 & 17.44482 & 0 \\
2 & 1.114931 & 82.67845 & 17.24562 & 0.07593 \\
3 & 1.115256 & 82.63117 & 17.23962 & 0.129215 \\
4 & 1.1153 & 82.62774 & 17.24106 & 0.131196 \\
5 & 1.115305 & 82.62751 & 17.24109 & 0.131406 \\
6 & 1.115305 & 82.62750 & 17.24109 & 0.131408 \\
7 & 1.115305 & 82.62750 & 17.24109 & 0.131408 \\
8 & 1.115305 & 82.62750 & 17.24109 & 0.131408 \\
9 & 1.115305 & 82.62750 & 17.24109 & 0.131408 \\
10 & 1.115305 & 82.62750 & 17.24109 & 0.131408 \\
11 & 1.115305 & 82.62750 & 17.24109 & 0.131408 \\
12 & 1.115305 & 82.62750 & 17.24109 & 0.131408 \\
13 & 1.115305 & 82.62750 & 17.24109 & 0.131408 \\
14 & 1.115305 & 82.62750 & 17.24109 & 0.131408 \\
15 & 1.115305 & 82.62750 & 17.24109 & 0.131408 \\
\hline
\end{tabular}

Cholesky Ordering: DPBIR DPROD DCANON 


\section{Anexo 4}

\section{Autocorrelación Modelo VAR 1}

\begin{tabular}{|c|c|c|c|}
\hline \multicolumn{4}{|c|}{$\begin{array}{l}\text { Equation: } \mathrm{D}(\mathrm{PBIR})=\mathrm{C}(1) * \mathrm{D}(\mathrm{PBIR}(-1))+\mathrm{C}(2) * \mathrm{D}(\mathrm{PROD}(-1))+\mathrm{C}(3) \\
\quad * \mathrm{D}(\mathrm{CANON}(-1))+\mathrm{C}(4)+\mathrm{C}(5) * \mathrm{DICOT}\end{array}$} \\
\hline \multicolumn{4}{|c|}{ Observations: 24} \\
\hline R-squared & 0.11542 & Mean dependent var & 190830.7 \\
\hline Adjusted R-squared & -0.070807 & S.D. dependent var & 414989.6 \\
\hline S.E. of regression & 429430.5 & Sum squared resid & $3.50 \mathrm{E}+12$ \\
\hline Durbin-Watson stat & 2.040063 & & \\
\hline \multicolumn{4}{|c|}{ Equation: $\mathrm{D}(\mathrm{PROD})=\mathrm{C}(6) * \mathrm{D}(\mathrm{PBIR}(-1))+\mathrm{C}(7) * \mathrm{D}(\mathrm{PROD}(-1))+\mathrm{C}(8)$} \\
\hline \multicolumn{4}{|c|}{$* \mathrm{D}(\mathrm{CANON}(-1))+\mathrm{C}(9)+\mathrm{C}(10) * \mathrm{DICOT}$} \\
\hline \multicolumn{4}{|c|}{ Observations: 24} \\
\hline R-squared & 0.021911 & Mean dependent var & -0.015 \\
\hline Adjusted R-squared & -0.184002 & S.D. dependent var & 1.017683 \\
\hline S.E. of regression & 1.10736 & Sum squared resid & 23.29866 \\
\hline Durbin-Watson stat & 1.918336 & & \\
\hline \multicolumn{4}{|c|}{ Equation: $\mathrm{D}(\mathrm{CANON})=\mathrm{C}(11) * \mathrm{D}(\mathrm{PBIR}(-1))+\mathrm{C}(12) * \mathrm{D}(\mathrm{PROD}(-1))+\mathrm{C}(13)$} \\
\hline \multicolumn{4}{|c|}{$* \mathrm{D}(\mathrm{CANON}(-1))+\mathrm{C}(14)+\mathrm{C}(15) * \mathrm{DICOT}$} \\
\hline \multicolumn{4}{|c|}{ Observations: 24} \\
\hline R-squared & 0.17321 & Mean dependent var & 2757374 \\
\hline Adjusted R-squared & -0.000851 & S.D. dependent var & 72600520 \\
\hline S.E. of regression & 72631400 & Sum squared resid & $1.00 \mathrm{E}+17$ \\
\hline Durbin-Watson stat & 1.85951 & & \\
\hline
\end{tabular}

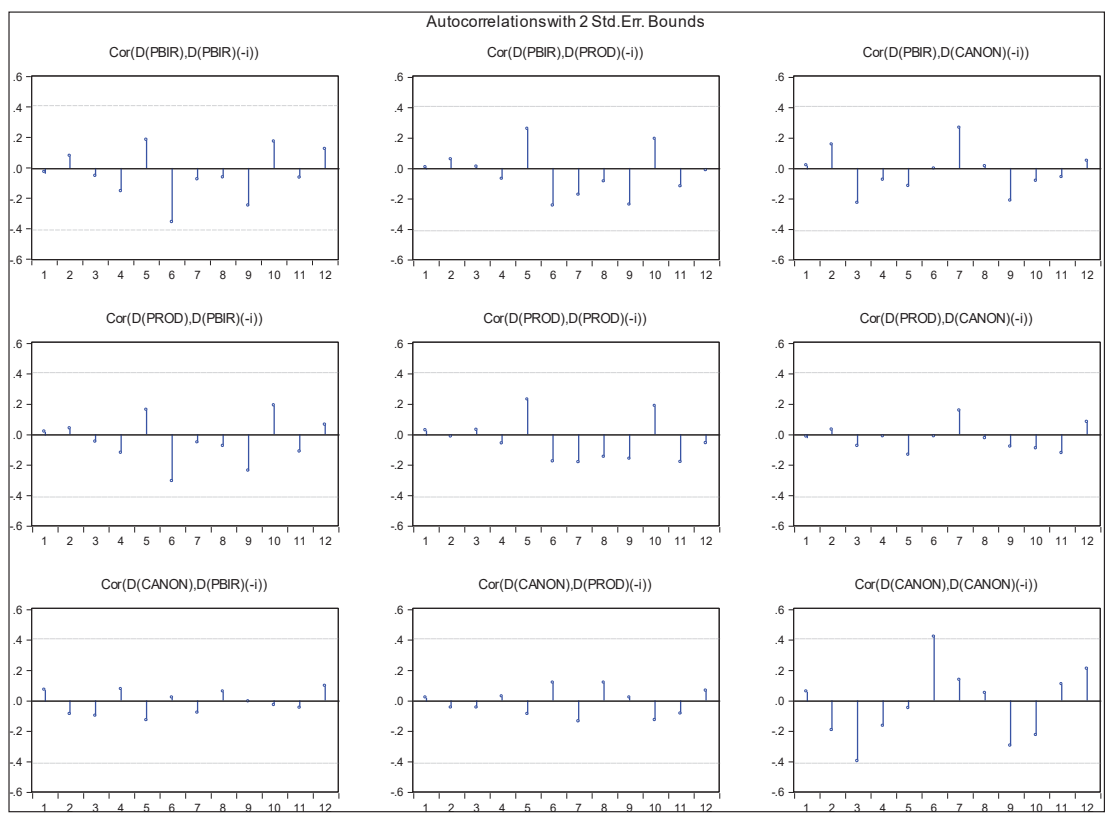


Anexo 5

Normalidad Modelo VAR 1

System Residual Normality Tests

Orthogonalization: Cholesky (Lutkepohl)

Null Hypothesis: residuals are multivariate normal

Date: 04/27/20 Time: 08:56

Sample: 19962019

Included observations: 24

Component Skewness Chi-sq df Prob.

$\begin{array}{lll}1 & -0.235725 & 0.222266\end{array}$

$2 \quad 0.070153 \quad 0.019686$

$1 \quad 0.6373$

3

$3 \quad-0.77592 \quad 2.408206$

$1 \quad 0.8884$

Joint

2.650157

1

0.1207

$3 \quad 0.4488$

Component

Kurtosis

Chi-sq

df

Prob.

$1 \quad 2.891223 \quad 0.011832$

1

0.9134

$2 \quad 2.060041$

0.883524

1

0.3472

3

3.24059

0.057884

1

0.8099

Joint

0.95324

3

0.8126

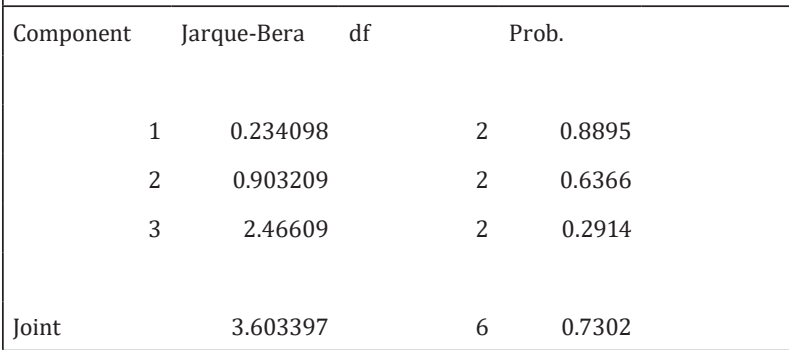




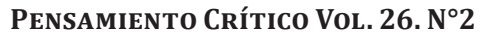

\section{Anexo 6}

Descomposición de Varianza VAR 2

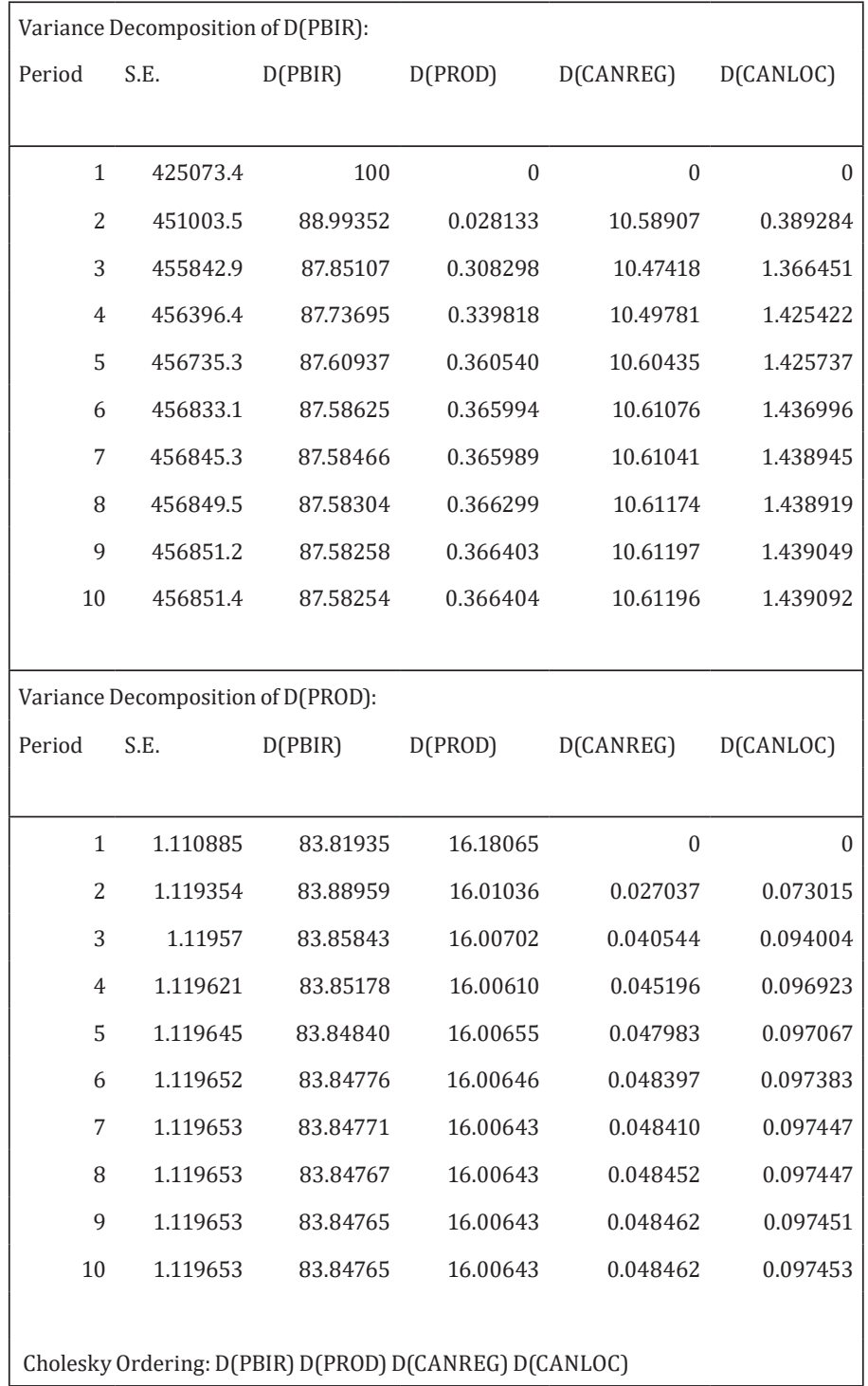




\section{Notas al final}

1 Artículo de investigación científica

2 Economista y Magister en Finanzas por la Universidad Nacional de la Amazonía Peruana. Doctor en Gestión Económica Global por la Universidad Nacional Mayor de San Marcos. Docente universitario. (e-mail: alexci.chong@ unmsm.edu.pe). Celular 965967938

3 Economista (UNP), estudios Maestría Economía (PUCP), Maestría y Doctorado en Política y Gestión del Desarrollo (Antwerpen University, Bélgica). Prof. FGAD-PUCP, Posgrado UNMSM. (wong.la@pucp.pe). Celular 985596520

4 Economista. Jefe de Departamento de la Escuela de Economía de la Universidad Nacional de la Amazonía Peruana. Decano del Colegio de Economistas de Loreto. (vladimir.chong@gmail.com). Celular 965605690

5 La curva de producción del petróleo sigue una curva gaussiana. Cuando la producción alcanza la meseta significa el punto máximo de producción de petróleo, punto a partir del cual la tasa de extracción comienza a disminuir.

6 Ya en el siglo XVII Holanda un país de bajos recursos naturales eclipsó a España a pesar de los inmensos flujos de oro y plata de las colonias españolas en el nuevo mundo. Del mismo modo, durante los siglos XIX y XX Suiza y Japón, así como los tigres del Asia, países con escasos recursos estuvieron por delante de Rusia, México, Venezuela, Nigeria, países con abundante recurso natural e incluso, quebraron.

7 Agregamos que esto es ambiguo en la teoría económica, ya que una apreciación del TCR que tiene como contrapartida una depreciación de la moneda local, abarata los productos de exportación.

8 Para mayores luces, la apreciación de la moneda holandesa, seguido después del boom de las exportaciones de gas, causó inflación, que a su vez provocó reducciones en la competitividad y rentabilidad en los sectores manufacturas y servicios.

9 El ítem V discusión, amplía las consideraciones sobre el modelo VAR.

10 Tratándose de un modelo VAR el choque (impulso o innovación) se traduce a cada variable dependiente simultáneamente y ver su efecto en el sistema VAR.

$11 \mathrm{~A}$ fin de no caer en la trampa de las variables dummies Loreto representa el intercepto diferencial (Const) en el modelo de datos de panel de efectos fijos.

12 Debido a la naturaleza dinámica del modelo es sugerible que las ecuaciones estén sonreindetiificadas el cual permite que los estimadores mantienen una combinación lineal de todas las combinaciones posibles en el modelo.

13 Extraído de consulta amigable del MEF en base a recursos determinados, canon y sobrecanon petrolero, devengados (Disponible en Fuentes / rubro / genérica del gasto) 